J. Lake Sci. (湖泊科学) , 2018, 30(6): 1489-1508

DOI 10. 18307/2018. 0602

(c) 2018 by Journal of Lake Sciences

\title{
沉积物一水界面污染物迁移扩散的研究进展”
}

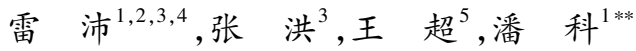 \\ ( 1 : 深圳大学高等研究院, 深圳 518060$)$ \\ (2: 深圳大学光电工程学院光电子器件与系统 (教育部/广东省) 重点实验室, 深圳 518060) \\ (3: 中国科学院生态环境研究中心环境水质学国家重点实验室,北京 100085) \\ ( 4 : 南京大学环境学院, 南京 210046) \\ (5:长江水资源保护科学研究所,武汉 430051)
}

摘 要: 污染物在沉积物一水界面的迁移扩散对研究其环境生物地球化学循环过程和评估水生态系统质量具有重要意 义. 本文回顾了关于沉积物一水界面的基本研究历程, 重点介绍沉积物一水界面的垂向结构以及扩散边界层 (DBL) 的作 用, 展示污染物在沉积物一水界面的多维度分布 (一维垂向、二维平面和三维立体) 及其在沉积物一水界面的扩散过程, 详 细总结影响污染物在沉积物一水界面迁移扩散的环境因素 (包括温度、溶解氧或氧化还原条件、 $\mathrm{pH}$ 值、离子强度或盐度、 沉积物组成、共存污染物、溶解性有机质、水动力条件、生物扰动、微生物以及其他因素), 讨论当前关于沉积物一水界面污 染物扩散通量估算几种方法的优缺点, 最后, 对沉积物一水界面污染物扩散研究在未来发展需要关注的几个方面进行了 展望.

关键词: 沉积物一水界面;迁移扩散;扩散通量;污染物

\section{Migration and diffusion for pollutants across the sediment-water interface in lakes: A re- view}

\section{LEI Pei ${ }^{1,2,3,4}$, ZHANG Hong ${ }^{3}$, WANG Chao ${ }^{5} \&$ PAN $\mathrm{Ke}^{1 * *}$}

(1: Institute for Advanced Study, Shenzhen University, Shenzhen 518060, P.R. China)

(2: Key Laboratory of Optoelectronic Devices and Systems of Ministry of Education and Guangdong Province, College of Optoelectronic Engineering, Shenzhen University, Shenzhen 518060, P.R.China)

(3: State Key Laboratory of Environmental Aquatic Chemistry, Research Center for Eco-Environmental Sciences, Chinese Academy of Sciences, Beijing 100085, P.R.China)

(4: School of Environment, Nanjing University, Nanjing 210046, P.R.China)

(5: Changjiang Water Resources Protection Institute, Wuhan 430051, P.R.China)

\footnotetext{
Abstract: Exploring migration and diffusion for pollutants across the sediment-water interface is important for understanding the biogeochemical cycles of pollutants and assessing the environmental quality of lake ecosystems. In this review, we reviewed studies on "sediment-water interface" in the past decades, and illustrated the vertical structures and function of diffusive boundary layer (DBL) of the sediment-water interface. Then, we presented the multi-dimensional distribution ( e. g. Distribution of 1-D verticality, 2-D plane, and 3-D space) of pollutants and diffusion process across the sediment-water interface. Also, we listed some key factors influencing diffusive process ( such as temperature, dissolve oxygen or redox potential, salinity or ionic strength, sediment composition, coexistence of pollutants, dissolved organic matter, hydrodynamics, bio-turbation, microorganism and other factors). Some recent advances in the measurements of the pollutant flux across the sediment-water interface were summarized. Finally, demands and challenges about the migration and diffusion for pollutants across the sediment-water interface in future research

* 中国博士后科学基金项目(2017M622782)、国家自然科学基金项目 (41676095) 和深圳市知识创新计划基础研究项 目 (JCYJ20160422153856130) 联合资助. 2018-03-18 收稿; 2018-05-02 收修改稿. 雷沛 (1989 ), 男,博士; E-mail: leipei29@163.com.

** 通信作者;E-mail: panke@ szu.edu.cn.
} 
are proposed.

Keywords: Sediment-water interface (SWI) ; migration and diffusion; diffusive flux; pollutants

沉积物一水界面是水生态系统中最重要的界面之一, 是物质参与环境地球化学循环和生物耦合的 “热 区”. 在微生物参与下, 沉积物一水界面附近物质发生一系列的物理化学及生物反应( 如迁移、转化、吸附/解 吸、扩散、掩埋以及生物扰动等), 是调节和控制沉积物和水体之间物质交换和输送的重要途径 ${ }^{[1]}$. 沉积物中 大量溶解性物质主要以孔隙水作为媒介通过扩散向上覆水体迁移, 从而影响上覆水水质; 上覆水体通过生 物同化、物理沉降和扩散等过程为界面反应提供物质基础. 认识沉积物一水界面结构以及污染物在沉积物一 水界面的扩散过程,对探讨污染物环境行为具有重要的理论和现实意义. 本文总结了关于 “沉积物一水界 面” 的基本研究历程, 简要介绍了沉积物一水界面的结构与作用, 以及物质在沉积物一水界面的扩散过程, 详 细总结了影响沉积物一水界面污染物迁移扩散的环境因素, 讨论了当前关于沉积物一水界面污染物扩散通 量估算几种方法的优缺点, 以期为水体内源污染控制和风险辨识提供科学支撑和理论依据.

\section{1 沉积物一水界面的基本研究历程}

近 30 年来,关于 “沉积物一水界面” 文献报道的数量呈线性增加 (图 1b), 而发表文献的被引频次几乎呈 指数增长 (图 1c). 就研究领域而言, 在环境科学与生态领域中开展的研究一直是 “沉积物一水界面” 最为关 注的热点; 此外, 在海洋学、水资源、公共环境与职业健康等方面也有一定的关注 (图 1d). “沉积物一水界 面”, 已成为探索污染物环境生物地球化学行为的重要 “热区”, 对研究水体污染物迁移转化与风险评估方面 具有重要意义.

在时间发展上,人们对沉积物一水界面污染物的关注,随水体污染类型以及新方法、新技术的研发、应用 而逐步发展, 从营养盐、重金属、有机污染物等单一指标的监测逐步过渡到多种污染物共存形成的复合污染 效应的关注 (图 1a). 早在 1855 年, 菲克扩散定律的提出为研究界面物质扩散奠定了理论定量计算基础, 也 是研究沉积物一水界面污染物扩散通量最重要的依据之一. 随后, 国内外学者对沉积物一水界面逐步开展了 在物理学、化学、微生物学以及生物地球化学等多学科交叉研究. 1960s, 世界范围内的湖泊、水库出现了严 重的富营养化问题, 学者们开始关注沉积物一水界面营养盐的吸附/解吸, 以及环境因子对该过程产生的影 响, 并意识到内源也是湖库等水体污染负荷的重要贡献来源之一. 1976 年, 平衡渗析装置 (Peeper) 开始原位 应用于沉积物孔隙水中营养盐、重金属无机污染物平衡浓度的测定, 从而能获取厘米级高分辨率的浓度剖 面, 为认识沉积物一水界面污染物的浓度分布与扩散通量估算提供了技术支撑 ${ }^{[2-3]} .1980$ 年, 原位箱 (Chamber) 用于研究污染物在沉积物一水界面的交换作用及扩散通量 ${ }^{[4]} .1990$ 年, 溶解微电极的应用使人们 对沉积物一水界面多维结构有了新的认识 ${ }^{[5]}$. 进人 21 世纪以后, 学者们不断地提出和推进有关沉积物一水 界面的一些新技术、新方法, 如选用垂向分辨率达 $2 \mathrm{~mm}$ 的 Peeper 研究沉积物一水界面的铁磷耦合作用机 制 ${ }^{[6]}$, 基于平面光极技术、薄膜扩散梯度技术开展沉积物一水界面理化性质和污染物迁移性的研究 ${ }^{[7-8]}$, 利用 微仿和微采样系统 ( missy) 联合超滤和浊点萃取提取以毫米的分辨率来区分沉积物孔隙水中胶体态和溶解 性组分 ${ }^{[9]}$ 等等, 这些都提升了人们对沉积物一水界面污染物高分辨率、多维度分布、多污染物耦合作用机制 的认识,为进一步探索污染物的环境行为提供了良好的技术支撑.

1990s 开始, 水体中有机污染物的风险问题日渐显现, 疏水性有机污染物 (如 PAHs 等) 在沉积物一水界 面分布及其吸附等环境行为也逐步受到重视 ${ }^{[10-11]}$; 进人 21 世纪, 沉积物一水界面疏水性有机污染物扩散通 量的估算及其风险评估受到了广泛关注, 如 Lick 探讨了分子扩散、生物扰动以及地下水交换对界面有机污 染物扩散通量的影响 ${ }^{[12]}$. 随后, 水体被动采样技术的研发及应用很好地解决了界面有机物扩散通量估算的 难题. 2010 年, Eek 等设计一套以半渗透膜为吸收材料的原位箱用来测定沉积物一水界面 PAHs 和多氯联苯 (PCBs) 扩散通量, 并对该通量计算方法进行了修正 ${ }^{[13]} .2013$ 年, 中国科学院广州地球化学研究所曾永平团 队研发了沉积物一水界面原位被动采样装置, 开展原位应用且通过运用数学模型来拟合获取沉积物一水界 面双对氯苯基三氯乙烷 (DDTs) 浓度分布剖面, 并由此确定了目标物的扩散通量方向与强度 ${ }^{[14]}$, 为认识此类 污染物的环境行为和生态风险提供了良好的技术手段. 
水体沉积物是多种污染物的共同 “蓄积库”, 众多无机、有机污染物组成了复杂的多相共存的混合体系, 其中, 污染物的生物、生态效应是多种污染物及自然物质发生相互作用后形成的长期综合效应 ${ }^{\left[{ }^{[15]}\right.}$. 为探索 污染物的“复合污染” 效应, 近几年, 部分学者开展了复杂环境基质中不同类别污染物同步前处理与测定等 创新性探索 ${ }^{[16-17]}$, 为此方向的推进提供了有力的支撑. 如基于平衡渗析和 LDPE 为吸附相的水体无机/有机 污染物同步被动采样装置, 可获取高分辨率沉积物一水界面无机/有机污染物自由溶解态浓度剖面, 并由此 计算沉积物一水界面扩散通量, 从而识别了沉积物对不同类别污染呈现的 “源”或 “汇” 的作用 ${ }^{[18]}$. 在 “复合 污染” 效应的背景下, 无机/有机污染物同步采样和分析是沉积物一水界面研究的一个重要方向, 未来研究中 应注重方法的标准化、原位应用可行性和生态毒理效应耦合等方面 ${ }^{[19]}$.
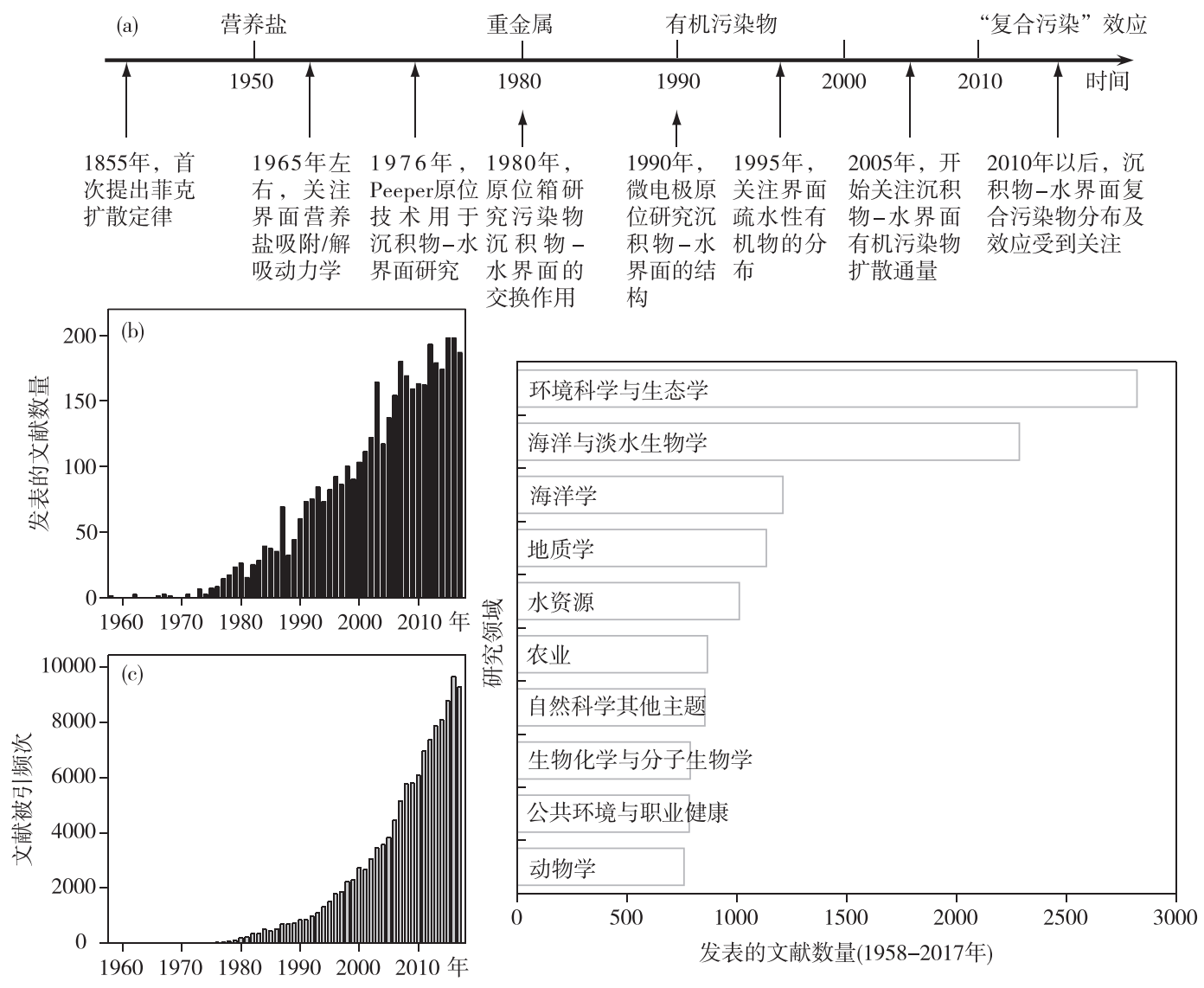

图 1 近 60 年 $(1958-2017$ 年) 来有关“沉积物一水界面” 的文献报道情况

( (b)、(c)、(d) 数据来自于 Web of Science(http://isiknowledge.com/), 以“sediment-water interface”为关键词开展的主题检索)

Fig.1 Publications about "sediment-water interface" in the past 60 years (1958-2017)

\section{2 沉积物一水界面结构及污染物的多维度分布}

\section{1 沉积物一水界面的垂向结构}

界面是指两个或多个物相之间的分界面, 是一个既具体又抽象的概念. 水生态系统中沉积物一水界面 (Sediment-water interface, SWI), 宏观上是指沉积物相和上覆水相两种固液体介质因物理接触而形成的 “界 面”或过渡区,但实际上沉积物相与水相之间并不存在一个接触的 “面” ; 从微观角度来看, 沉积物一水界面 是两种介质在一定空间上的相互渗透和包含, 从而使 “界面” 在垂向上进行拉伸, 使界面有了立体尺度. 由于 
重力作用, 密度大于水的颗粒沉于水底, 在早期成岩和物理压实作用下, 形成含水量和孔隙度不等的沉积 层,并随沉积作用使沉积物一水界面在垂向上有所延展 ${ }^{[20]}$.

沉积物一水界面的垂向结构与水流的摩擦作用密切相关, 水流的平均流速和湍流程度会决定界面垂向 各层的厚度与特性 ${ }^{[21]}$. 在表层沉积物上部区域, 水体平均速度的增加随高度呈对数上升, 该层称为对数层 (Logarithmic layer). 在水底界面附近,物质以黏滞迁移为主,该层称为黍滞亚层 (Viscous sublayer); 尽管该层 非常薄 (厘米级), 但对物质迁移的阻力最大, 溶质和热量在该层混合相对充分, 剪应力也相对恒定. 在最接 近界面处, 即使存在波浪形成的重力流和浴流的影响下, 沉积物表面上仍然会有约几百微米厚的水膜, 膜内 物质的分子扩散超过浴流扩散,该层被称为扩散边界层 (Diffusive boundary layer, DBL). 自水层垂直向下就 将穿过理论上没有 “厚度” 的沉积物一水界面, 进人由沉积颗粒组成的多孔性布林克曼层 (Brinkman layer), 该层是可流动和多孔介质复合的广义边界层, 呈多孔渗水状态, 容易受到湍流的影响, 形成一种水压力驱动 的达西流( Darcy flow) ${ }^{[20]}$ (图 2).
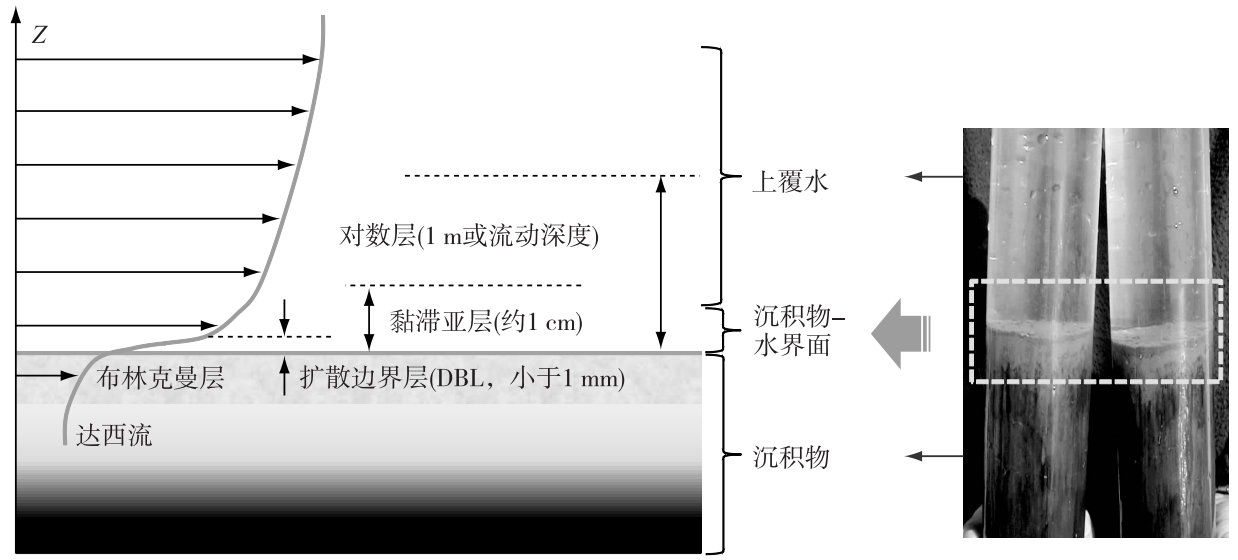

图 2 沉积物一水界面垂直结构示意图 (改自文献 $[21]$ )

Fig. 2 Diagrammatic sketch of vertical structure of sediment-water interface

沉积物一水界面是水和沉积物相互交叉的区域, 它们之间没有十分明显的分界线. 沉积物和上覆水之间 的物质交换对水生态系统中一系列生物化学过程起着重要作用,但是 DBL 限制了这一交换,成为沉积物一 水界面扩散通量的主要瓶颈 ${ }^{[22]}$. DBL 是沉积物上很薄的一层 (一般情况下其厚度均会小于 $1 \mathrm{~mm}$ ), 当物质 从潮流底层输送进人 DBL 后, 分子扩散会取代湍扩散成为控制垂直输运的主要因素. 这是因为, 在 DBL 下 方由于沉积物表面黏滞力使得物理扰动作用不显著, 该层中物质交换的主要机制为分子扩散 (浴流扩散接 近于零), 因此可认为 DBL 中物质浓度梯度呈现线性变化. 事实上, DBL 上边界被定义为沉积物一水界面分 子扩散系数 $\left(D_{\text {molecular }}\right)$ 等于 $D_{\text {eddy }}$ 时对应的厚度 ${ }^{[5]}$. 在界面垂向上, 恒定温度下特定溶质的 $D_{\text {molecular }}$ 保持恒定, 而浴流扩散系数从上覆水向沉积物呈指数衰减 (如 $D_{\text {eddy }} \propto Z^{3.38}, Z$ 为沉积物一水界面的高度) 并在 DBL 中快 速衰减至零(图 3).

水底动力条件会显著影响 DBL 的厚度, 进而改变沉积物一水界面物质的扩散通量, 因此, 准确测定 DBL 厚 度是有效获取界面扩散通量的关键前提. Gundersen 等首先在实验室开展了 DBL 的系统研究, 其利用溶解氧微 电极 (垂向分辨率达 0.05 0.1 mm) 观测 DBL 垂向结构, 发现 DBL 厚度在 0.2 1 mm 之间, 并受上覆水流速和 沉积物地形粗糧度的影响 ${ }^{[5]}$. 然而, 获取沉积物一水界面 $1 \mathrm{~mm}$ 内高分辨率物质含量变化, 通过拟合确定 DBL 的厚度并计算其扩散通量, 是目前的技术难点之一. 借鉴室内实验经验, Reimers 等利用溶解氧微电极在深海 对 DBL 进行现场观测, 垂直分辨率可达 $1 \mathrm{~mm}^{[23]}$, 虽然这远不能满足 DBL 研究的高分辨率要求, 但也显著提高 了界面扩散通量的计算准确性. 需要指出的是, 不同污染物在 DBL 中的分布与溶解氧可能存在一定的差异, 因 此,多物质同步测定微电极的研发和应用也是未来沉积物一水界面研究中需要重点发展的方向.

当然,对于一般的环境地球科学研究, 由于技术的限制我们通常无法获取 DBL 中高分辨率的污染物浓 


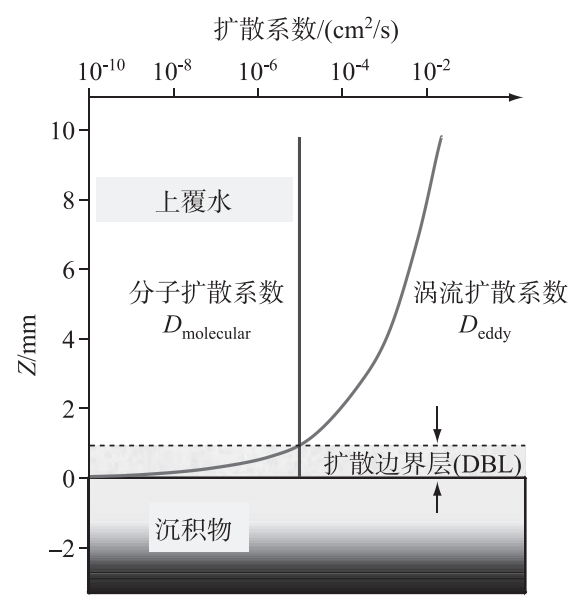

图 3 沉积物一水界面扩散边界层 $(\mathrm{DBL})$ 厚度的确定 $\left(D_{\text {molecular }}\right.$ 等于 $D_{\text {eddy }}$ 时对应的厚度,改自文献 $\left.[5]\right)$

Fig.3 The upper limit of the diffusive boundary layer (DBL) is the height above the sediment at which $D_{\text {molecular }}$ equals $D_{\text {eddy }}$

度梯度变化,但可以采用一些简化处理来得到估算的结果. 例如,我们可利用主动采样方法将沉积物样柱进 行分层、离心, 获取孔隙水并测定其中污染物的浓度剖面,并根据浓度梯度法(见 5.1 节) 来计算该物质在沉 积物一水界面的扩散通量. 虽然这种方法获取的分辨率非常有限 (如 $0.5 \mathrm{~cm}$ ), 仍然远超过 DBL 的实际厚度, 但此时我们可近似认为该范围内污染物的浓度梯度是呈线性变化的, 由此估算得到的扩散通量在宏观上也 能提供一定信息和指导. 此外, 采用相对较高的分辨率 (如亚厘米级和毫米级) 的被动采样技术 (如薄膜扩散 梯度技术 DGT、Peeper、沉积物一水界面原位被动采样装置等) 获取沉积物一水界面无机/有机污染物的浓度 剖面也是一个不错的替代方法.

\section{2 沉积物一水界面污染物的多维度分布}

沉积物一水界面只是一个相对稳定的结构, 当水底边界层内流体摩擦力形成的剪切作用大于起动切应 力后, 表层沉积物就将被扰动产生再悬浮, 使沉积物一水界面受到破坏, 在平面上呈一定程度的整体下移. 而 且, 自然水生态系统 (如湖泊) 是一个具有高度生物活性的特殊环境, 水体中的微小颗粒物不断沉降并进人 沉积物, 在沉积物一水界面发生沉积和早期成岩作用; 并且在微生物和胞外酶的作用下, 有机质发生降解和 矿化,直接影响碳、氮、磷、硫、铁及锰等生源要素的生物地球化学过程.

自然水生态系统沉积物的孔隙会被水体充盈 (即孔隙水, Pore water), 溶解态污染物、胶体或微小颗粒物 可通过孔隙水渗透进人沉积物. 而沉积物中的污染物也可以进人孔隙水并在适当条件下通过扩散进人上覆 水体. 沉积物孔隙中的通畅性是影响物质向沉积物迁移的重要因素. 沉积物中的孔隙是呈分支网状的立体 三维结构分布的, 这就决定了溶质以及水分子在沉积物中不会以直线的方式进行迁移 ${ }^{[20]}$. 因为沉积物具有 孔隙度 ( porosity, $\varphi$ ) 和绕曲度 (tortuosity, $\theta$ ) 效应, 所以沉积物孔隙水中物质的扩散通量在同等条件下要小 于自由水体 ${ }^{[24]}$.

沉积物一水界面的 “结构” 主要是通过界面物质 (污染物) 的分布来体现. 菲克扩散定律的提出对认识物 质在沉积物一水界面的迁移扩散提供了模型化的定量基础. 传统关于沉积物一水界面结构认识的实验手段 (对沉积柱进行分层) 主要是限定在厘米尺度, 自 1980s 逐步发展的溶氧微电极、被动采样技术、孔隙结构解 析技术等手段为人们研究溶解氧、磷酸盐、重金属、有机物等污染物在沉积物一水界面扩散迁移等环境行为 提供了定量分析手段,也推进了人们对沉积物一水界面结构的多维度理解和认识.

2.2.1一维垂向分布 由于水体 DBL 厚度一般只有 $0.2 \sim 1 \mathrm{~mm}$, 为了能准确测定 DBL 中物质浓度梯度并计算 界面扩散通量, 就需要至少在垂向上有 $50 \sim 100 \mu \mathrm{m}$ 的分辨率. 微电极被认为是有效地认识氧气、硫等物质 在沉积物一水界面垂向上特别是在 DBL 中浓度分布的有效手段. 微电极足够小 ( 尖端直径 $1 \sim 10 \mu \mathrm{m}$ ), 它们 能在沉积物一水界面 DBL 中直接进行超高分辨率 (可达 $20 \mu \mathrm{m}$ ) 的精细测定 ${ }^{[25]}$. 利用溶解氧微电极系统可 
精细地获取沉积物一水界面溶解氧含量的微小变化. 通过测定发现, 上覆水中的溶解氧维持在相对稳定的水 平 $\left(C_{w}\right)$, 当进人 DBL 后溶解氧的浓度开始逐渐、显著地呈线性下降, 由此可以确定 DBL 的厚度 $\left(z_{\delta}\right)$ 并准确 地计算通过沉积物一水界面的溶氧扩散通量 ${ }^{[22]}$ (图 4a). 开展微电极系统的原位应用是推进沉积物一水界面 研究的一个重要方向, 其中, 丹麦 Unisense 公司已成功研发了一系列用于测定沉积物一水界面、植物根际微 环境等商用微电极,为研究界面物质迁移转化提供了支撑.

1994 年, 薄膜扩散梯度技术 (DGT) 的发明实现了沉积物一水界面高分辨率(毫米级) 重金属离子浓度剖 面的获取 ${ }^{[26]}$. 随后, 该项技术被广泛地应用于磷酸盐 ${ }^{[27]}$ 、硫化物 ${ }^{[28]}$ (图 4b)、多种金属 ${ }^{[29]}$ (图 4d) 等污染物 在沉积物一水界面垂向浓度剖面分布, 极大地帮助了人们开展这些无机污染物在沉积物一界面的迁移转化、 生物可利用性评估、风险或毒性预测等研究 ${ }^{[30]}$. 与此同时, 被动采样技术的研发与应用,使得获取沉积物一 水界面低浓度但具有高毒性的疏水性有机污染物的浓度分布成为了可能 ${ }^{[14]}$ (图 4c), 推进了人们对沉积物一 水界面疏水性有机污染物的环境化学行为与生态风险的认识.

2.2.2 二维平面分布 通常对沉积物一水界面污染物浓度分布仅在垂向尺度表现出显著的梯度变化,而在其 他维度上我们一般假设其浓度为相对均匀分布, 但实际上, 沉积物本身是立体多孔隙结构, 这表明实际条件 下污染物也会存在其他维度的迁移扩散. 2002 年, Lewandowski 等设计一套基于平衡理论的二维的沉积物一 水界面高分辨率 $(9 \mathrm{~mm} \times 9 \mathrm{~mm})$ 渗透式被动采样器, 并在德国 Mullrose 湖开展原位应用, 获取了较好的界面 溶解性活性磷 $(\mathrm{SRP})$ 二维平面分布图 ${ }^{[31]}$. 结果显示, 沉积物孔隙水中 SPR 浓度表现出较高的水平, 并在水 平、垂直两个方向呈现较强的异质性, 结果也指示 “沉积物一水界面”也并非一条 “水平的线” (图 4e). 在最 接近沉积物一水界面处因为有较大的浓度梯度从而存在较大的扩散通量, 但是扩散呈现向四周发散方向而 非传统假设的单一方向扩散 (图 4f). 除此之外,一些学者也将 DGT 发展为二维尺度, 并结合成像技术对沉 积物一水界面中硫、磷以及重金属的分布及迁移转化等开展了许多原创性工作 ${ }^{[32-33]}$, 提升了人们对沉积物一 水界面多维尺度、多污染物耦合作用的认识.

2.2.3 三维立体分布 早在 1990 年, Gundersen 等就利用高分辨率 (垂向 $50 \mu \mathrm{m}$ 、水平方向 $200 \mu \mathrm{m}$ ) 溶氧微电 极测定海洋沉积物溶氧浓度, 由此计算了沉积物表层 $10 \mathrm{~mm}^{2}$ 范围内 DBL 厚度的空间分布, 并通过 $17 \times 17$ 测 量点的数据来绘制沉积物表面的三维立体地形图. 研究结果发现, 表层沉积物呈现 “沟敍纵横” 的形态 (图 $4 \mathrm{~g}$ ), 而且 DBL 厚度与沉积物表层地形呈现高度吻合, 计算表面沉积物粗䊅的表面面积比沉积物平坦的表面 积的有效面积要大 $88 \%$. 在考虑沉积物三维地形分布的基础上, 利用扩散模型计算的溶氧通量要比传统的 一维 (垂直方向) 计算的溶氧通量高 2.5 倍 ${ }^{[5]}$, 这说明传统一维结构的沉积物一水界面扩散通量的估算会显 著低估水体内源释放风险. 在沉积物三维立体孔隙结构方面, 2013 年, 中国科学院生态环境研究中心单保庆 团队通过自主研发的原位液氮快速冷冻固定沉积物技术, 结合树脂包埋的采样、制样, 并集成其他学科的相 关研究方法, 构建了一套包含样品采集与制备方法、数据获取方法、图像解译、参数计算等研究湖泊沉积物 三维物理结构的方法学, 揭示了与物质转化扩散密切相关的孔隙数量、体积分布层次、表面曲折程度、连通 程度等孔隙特征. 在典型浅水湖泊开展应用, 显示随深度的增加沉积物垂向上孔隙度减少、连通性变差、孔 隙表面越来越曲折/粗徙, 最显著变化发生在沉积物一水界面小于 $1 \mathrm{~mm}$ 范围以内 ${ }^{[34]}$ (图 $4 \mathrm{~h}$ ), 这些工作为沉 积物一水界面不同深度的三维立体孔隙结构提供了数字化的展示, 为精确化认识沉积物一水界面污染物迁 移扩散提供了基础.

\section{3 污染物在沉积物一水界面的扩散过程}

单个分子或离子在液相或气相环境中表现出不可预测的随机运动, 然而, 更多的分子或离子会倾向于 从浓度高的区域转移到浓度低的区域, 直至介质中物质的浓度达到均匀 (即处于平衡状态), 这种定向地物 质运动过程即为扩散 (Diffusion). 在平衡状态下, 虽然个别分子和离子继续维持随机性运动, 但是不会发生 物质扩散. 扩散过程可适用于物质或热量通过液相、气相甚至是固相的传递, 在本文的讨论主要限定在污染 物在沉积物孔隙水和上覆水垂直方向上的扩散. 对一个封闭的系统而言, 当不存在外部干扰、没有溶质补充 进人系统以及系统中的溶质不会产生或消耗时, 所有浓度梯度就会逐渐减小直至溶质均匀分布在系统中直 到平衡状态建立起来(图 5). 


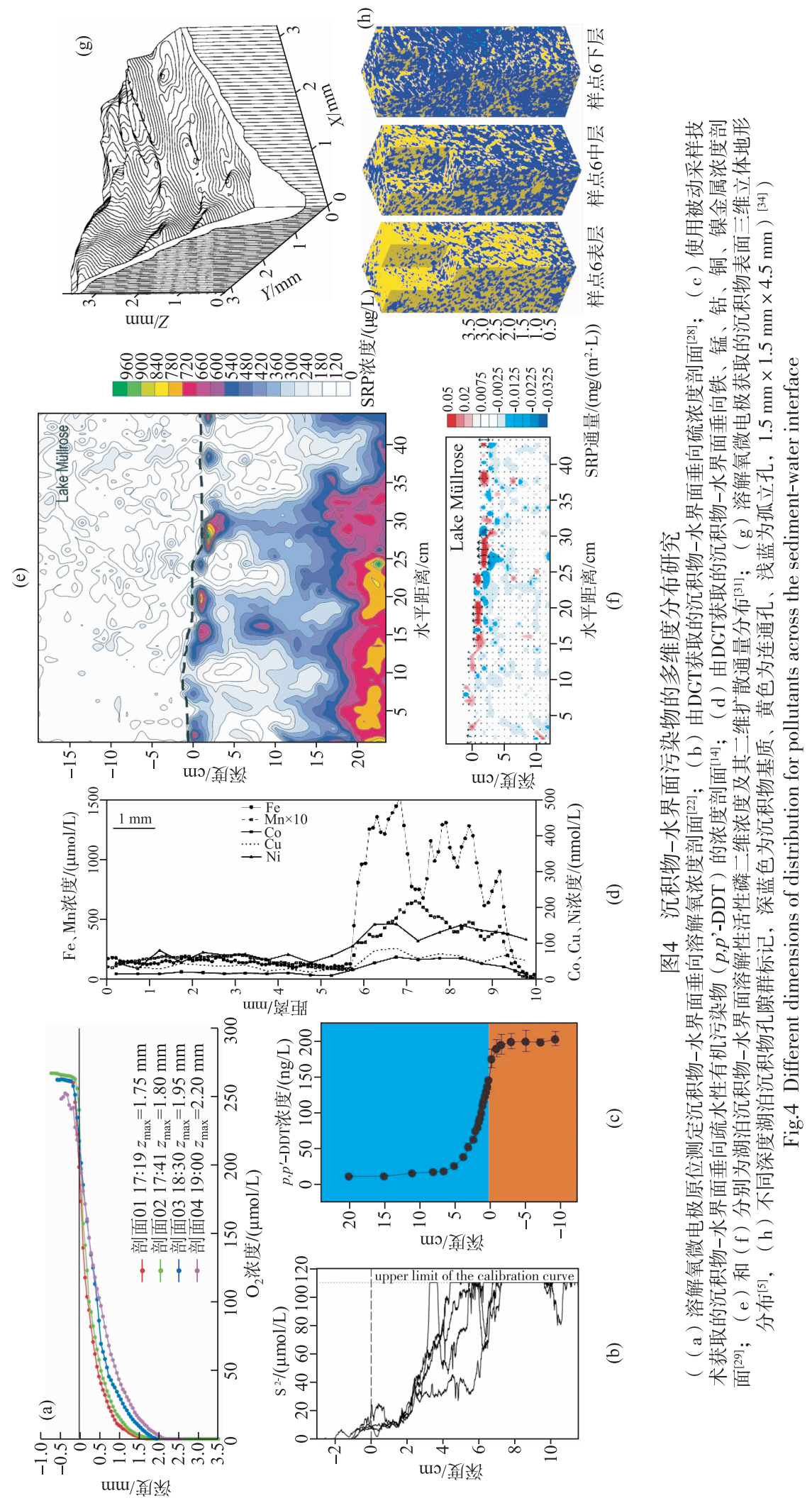




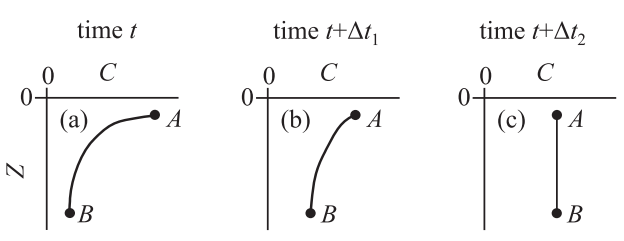

图 5 溶质的扩散过程

( ( a ) 和 (b) 显示了溶质随时间变化的非稳态 (即 $\left.\partial C_{(z, t)} / \partial z\right)$ 扩散下浓度梯度的减小, $C$ 为溶质浓度, $t$ 为时间, $z$ 为系统边界下的距离; 其中 $\Delta t_{1} \ll \Delta t_{2} ;(\mathrm{c})$ 显示系统处于平衡状态, 此时系统中溶质浓度相等)

Fig.5 The diffusive process of solutes in a system

分子扩散 (Molecular diffusion) 和涡流扩散 (Eddy diffusion) 是两种主要的扩散类型, 且均会影响沉积物一 水界面附近物质的运动. 由物质分子热运动而产生的扩散称为分子扩散; 分子扩散是当体系处于静止状态 时,溶质从高浓度区域向低浓度区域的单一方向运动. 浴流扩散是湍流流体中质点传递的主要方式, 是不同 微粒行走路径的不同而产生的扩散. 湍流流体中的质点沿各方向作不稳定的不规则运动, 于是流体内出现 旋涡, 旋浴的强烈混合所引起的物质传递比分子运动的作用大得多, 前者是以质点的规模而后者则是以分 子的规模进行的, 故浴流扩散的速率远大于分子扩散. 在湍流流体中也存在分子扩散, 但在大多数情况下分 子扩散可以忽略. 在不受干扰的沉积物孔隙水中不会发生浴流扩散因为此时孔隙水处于静止状态. 然而, 在 有外力干扰时就会发生浴流扩散, 如对流的外力 (例如浅水湖泊的风浪作用) 将水带人或带出沉积物、沉积 物受到突然挤压 (例如生物扰动) 或沉积物颗粒发生了再悬浮等 ${ }^{[35]}$.

溶质随机运动的结果可以用菲克扩散第一定律 (Fick's first law of diffusion) 来预测, 对于分子扩散和浴 流扩散, 通过沉积物一水界面的物质扩散通量与其浓度梯度呈正比,如下:

$$
J_{(z, t)}=-D \frac{\partial C_{(z, t)}}{\partial z}
$$

式中, $J_{(z, t)}$ 为物质扩散通量, 即单位时间单位面积通过物质的量 $\left(\mathrm{mg} /\left(\mathrm{m}^{2} \cdot \mathrm{d}\right) ; D\right.$ 为环境温度下溶质的传质系 数 $\left(\mathrm{cm}^{2} / \mathrm{s}\right) ; C$ 为浓度 $(\mathrm{mg} / \mathrm{L}) ; z$ 为垂直距离 (从沉积物一水界面的上边界开始, 随着沉积物深度的增加而增 加 $(\mathrm{cm}) ; t$ 为时间; $\partial C_{(z, t)} / \partial z$ 指对距离 $z$ 的一阶偏微分, 即浓度梯度 $(\mathrm{mg} /(\mathrm{L} \cdot \mathrm{cm})$, 浓度梯度是沉积物一水界 面物质扩散的主要驱动力.

从菲克扩散定量总结的公式 (1) 对分子扩散和浴流扩散均适用, 即公式使用分子扩散系数 $D_{\text {molecular }}$ 时, 获 得的通量为 $J_{\text {molecular }(z, t)}$; 当使用浴流扩散系数 $D_{\text {eddy }}$ 时, 得到的通量为 $J_{\text {eddy }(z, t)}$. 需要指出的是, 特定的温度下某 溶质的 $D_{\text {molecular }}$ 随着沉积物的深度和时间的变化保持恒定, 但 $D_{\text {eddy }}$ 随深度和时间发生较大的变化, 例如, 在水 溶液 $25^{\circ} \mathrm{C}$ 时, 无限稀释的溶质 $D_{\text {molecular }}$ 约为 $10^{-5} \mathrm{~cm}^{2} / \mathrm{s}$, 但 $D_{\text {eddy }}$ 可以从 0 (静止水体) 变化到非常大 (如瑞士 Zugersee 湖在极端风暴天气时可达到 $\left.10^{3} \mathrm{~cm}^{2} / \mathrm{s}\right)^{[36]}$.

在扩散通量实际计算中需要考虑沉积物孔隙度 $(\varphi)$ 和绕曲度 $(\theta)$ 效应对扩散系数的修正, $\theta$ 与 $\varphi$ 一般用 数学公式表示为 ${ }^{[24,37]}$ :

$$
\begin{aligned}
D_{\text {sed }} & =D_{\text {molecular }} / \theta^{2} \\
\theta & =1-2 \ln \varphi
\end{aligned}
$$

在实际计算中, $\varphi$ 是指沉积物体积中孔隙体积所占的比例, 可通过沉积物干湿重的差值进行简单地估 算. 式中, $D_{\text {sed }}$ 是指溶质在沉积物中的总体扩散系数, 显然 $D_{\text {sed }}$ 总是小于无颗粒物自由水体中的扩散系数 $\left(D_{\text {molecular }}\right)$.

当系统中溶质处于非稳态扩散且扩散唯一改变溶质浓度时, 可用菲克第二定律 (Fick's second law) 来描 述一定时间内系统中溶质浓度的变化率 ${ }^{[38]}$ :

$$
\frac{\partial C_{(z, t)}}{\partial t}=D \frac{\partial\left(\frac{\partial C_{(z, t)}}{\partial z}\right)}{\partial z}=D \frac{\partial^{2} C_{(z, t)}}{\partial z^{2}}
$$


式中, $\partial^{2} C_{(z, t)} / \partial z^{2}$ 指对距离 $z$ 的二阶偏微分, 同菲克第一定律一样, 菲克第二定律对涡流扩散和分子扩散也 都适用.

\section{4 影响污染物在沉积物一水界面迁移扩散的环境因素}

污染物在沉积物一水界面的迁移扩散过程十分复杂, 涉及吸附/解吸、溶解/吸收、络合/解离等一系列物 理化学作用以及生物氧化、还原、甲基化等生物地球化学过程, 除与污染物自身性质相关外, 相关环境因素 也会影响其迁移转化, 有些时候甚至会起到主要的支配作用. 很多学者在这方面开展了大量深人细致的研 究,并对其影响机理进行了细致的分析和探讨.

\section{1 温度}

一般当温度升高时, 沉积物对污染物的吸附能力降低, 沉积物中污染物通过孔隙水向上覆水的迁移会 增强. 温度对污染物在沉积物一水界面迁移的影响主要包括物质的传质系数、吸附能力、分配系数等方面, 如 研究发现夏季温度高时多氯联苯 (PCBs) 从沉积物向水体的迁移水平比冬季要高 ${ }^{[39]}$; 颗粒物对多环芳烃的 吸附比例随温度升高时呈显著增加趋势 ${ }^{[40]}$; 当温度从 $25^{\circ} \mathrm{C}$ 降低到 $2^{\circ} \mathrm{C}$ 时, 颗粒物对菼葱 (Flua) 的吸附分配 系数 $\left(K_{\mathrm{p}}\right)$ 升高了 $13 \%$,并推测这可能是由于温度的变化影响了其水溶解度 ${ }^{[41]}$.

\section{2 溶氧或氧化还原条件}

沉积物一水界面的溶解氧含量直接决定了有机质的矿化速率、功能性微生物的丰度及活性, 继而影响着 界面的物质循环过程. 当沉积物中溶解氧含量增加时, 硝化反应速率增加, 导致硝态氮、亚硝态氮交换速率 增加, 铵态氮交换速率降低 ${ }^{[42]}$. 缺氧条件下湖泊沉积物无机采的原位甲基化以及向上覆水甲基录的扩散都 会增加, 使底部水体中出现较高浓度的甲基永, 并通过垂直方向上的混合、平流和对流来影响其在表层水中 的分布 ${ }^{[43-44]}$. 氧化还原条件可改变重金属的形态和稳定性, 从而直接影响其迁移、释放 ${ }^{[45]}$. 氧化环境下重金 属多被铁锰水合氧化物固定,但若进人还原环境, 铁锰氧化物会被还原而使重金属释放出来 ${ }^{[46]}$. 还原性沉 积物中氧化还原电位增加能够加快硫化物的氧化, 并同时减少有机物质的含量, 从而加速吸附金属或螯合 金属的释放, 如 Cd 与有机硫的结合态一般认为是一种相对稳定的重金属形态, 但当沉积物氧化还原电位增 加时, 该形态含量会从 $65 \%$ 降至 $30 \%$, 生成迁移性更好的形态 ${ }^{[47]}$.

\section{$4.3 \mathrm{pH}$ 值}

一般认为, 高 $\mathrm{pH}$ 值能够增加重金属的吸附、沉淀, 而低 $\mathrm{pH}$ 值条件往往会减弱重金属的吸附, 从而缩短 沉积物中重金属的滞留 ${ }^{[48]}$. 研究表明, 低 $\mathrm{pH}$ 值可减少黏土颗粒、有机质以及 $\mathrm{Fe}-\mathrm{Mn}-\mathrm{Al}$ 氧化物等的表面负 电荷. 也有研究认为 $\mathrm{pH}$ 值的降低能促进碳酸盐和氢氧化物的溶解, 主要是由于 $\mathrm{H}^{+}$的竞争增加了金属离子 的解吸 ${ }^{[49]} . \mathrm{pH}$ 值对疏水性有机污染物迁移的影响主要是改变沉积物有机质结构及其溶解性. 在低 $\mathrm{pH}$ 值条 件下, 有机质中激发态半奎宁的松弛时间变长, 有机质呈分子状态, 可以保护疏水性位点, 有利于疏水性有 机污染物的吸附 ${ }^{[50]}$, 研究发现有机氯农药在沉积物中的吸附量随着 $\mathrm{pH}$ 值的增大而减小 ${ }^{[51]}$.

\section{4 离子强度或盐度}

在研究海洋、人海口、海湾等水生态系统时, 水体的离子强度或盐度对污染物尤其是重金属、有机物的 迁移产生重要影响. 一般情况下, 离子强度的增加会使沉积物有机质会发生团聚, 疏水性有机污染物的解吸 降低, 从而吸附增加 ${ }^{[52]}$, 但这种作用可能与体系中加人离子强度的量有关. 罗雪梅等研究发现向沉积物体 系中加人一定强度的 $\mathrm{Ca}^{2+}$ 时, $\mathrm{Ca}^{2+}$ 可中和沉积物中腐殖酸带的负电荷, 从而降低腐殖酸分子之间的静电排斥 作用, 腐殖酸也会卷曲变小进而发生团聚, 从而促进了腐殖质的胶束化和吸附能力较强的腐殖酸聚集体的 形成, 进而加大了吸附剂对吸附质的吸附; 但是, 当 $\mathrm{Ca}^{2+}$ 进一步增加时, 腐殖酸会继续团聚且相互分子之间 的疏水力增强, 会形成一种具有疏水内外表面的球型坚硬胶体, 此时腐殖酸就会絮凝或沉淀, 导致腐殖酸上 的疏水位很难以被有机物接近, 从而降低了吸附作用 ${ }^{[53]}$.

疏水性有机污染物在水中的溶解度会随着盐度的增加而减少, 即 “盐析效应” (Salting-out effect), 这一 现象可以用定量公式来描述 ${ }^{[41]}$ :

$$
\lg S_{\mathrm{w}, \text { salt }}=\lg S_{\mathrm{w}}-K_{\text {setch }}[\text { salt }]
$$

式中, $S_{\mathrm{w}, \text { salt }}$ 和 $S_{\mathrm{w}}$ 分别是污染物在咸水和纯水中的溶解度, $K_{\mathrm{sect}}$ 为盐度常数, 在一定温度下保持恒定, 如海水 
中芴 (Fluo) $K_{\text {setch }}$ 值范围一般为 $0.20 \sim 0.32 \mathrm{~L} / \mathrm{mol}^{[36]}$. 在此基础上, 得出 “盐析效应”对有机污染物分配系数 影响 $^{[41]}$ :

$$
\lg K_{\mathrm{p}, \text { salt }}=\lg K_{\mathrm{p}}+\alpha \cdot K_{\mathrm{setch}}[\mathrm{salt}]
$$

式中, $K_{\mathrm{p}, \text { salt }}$ 为在盐溶液中的分配系数, $\alpha$ 为常数,一般 PAHs 约为 $0.93^{[54]}$. 因此, 当在海水环境中应用被动采 样技术来测定有机污染物浓度时, 需要对其分配系数进行盐度校正.

\section{5 沉积物组成}

沉积物中有机质组成、黑炭含量或组分, 粒径结构等对污染物迁移和分配产生了重要的影响 ${ }^{[55]}$. 沉积 物中胡敏酸含有羧基和酚基官能团, 富里酸含有较多的羧基、羟基等官能团, 胡敏酸的芳香性以及对 PAHs 增溶效果均要强于富里酸, 而胡敏素主要是与沉积物中的矿物部分结合的腐殖质 ${ }^{[56]}$. 沉积物孔隙水中有机 污染物浓度在很大程度上取决于固液分配, 并与沉积物组成尤其是黑炭组分密切相关. 尽管沉积物中黑炭 占比一般小于 $1 \%$, 但它却吸附了沉积物中高达 $90 \%$ 的污染物 ${ }^{[57]}$; 同时由于它比非定型炭对疏水性有机物 的亲和力高 $10 \sim 100$ 倍, 从而会增加有机污染物在沉积物中的扩散阻力, 使其解吸受到限制 ${ }^{[11]}$. 当然, 沉积 物中颗粒物粒径大小对污染物的吸附、解吸及迁移存在一定的影响, 小粒径颗粒物具有更大的比表面积以 及更高的离子交换活性, 因此其对重金属的吸附能力也更强 ${ }^{[58]}$; 另有学者指出, 粗颗粒物中较大的石英矿颗 粒因具有三角或六角的光滑晶体表面, 无法为菌类提供足够的交互界面, 从而导致体系中氧化硫的含量降 低、重金属的溶解度增加 ${ }^{[59]}$.

\section{6 共存污染物}

在实际沉积物一水界面环境中, 多种有机、无机污染物组成了复合污染体系, 共存污染物之间复杂的交 互作用对污染物的迁移转化及环境效应, 特别是有机污染物界面过程会产生重要影响 ${ }^{[15]}$. 环境介质中许多 共存的有机污染物之间会存在竞争吸附作用,一般而言, 极性有机物竞争能力强于非极性有机物 ${ }^{[60]}$. Yu 等 研究表明共存萘、菲浓度的增加会显著降低沉积物中 $17 \alpha$-乙炔雌二醇的浓度及其在环境中的滞留性 ${ }^{[61]}$. 金 属阳离子可显著促进胡敏酸对多环芳烃的吸附, 抑制多环芳烃的解吸, 可能是由于金属阳离子促进胡敏酸 的絮凝, 改变有机碳的化学结构, 从而影响多环芳烃的界面反应 ${ }^{[62]}$. 表面活性剂也是环境中大量存在的有 机物之一, 它不但能影响沉积物性质, 具有较强的 “增溶” 作用 ${ }^{[63]}$. 如 Tritori-100 在表面活性剂临界胶束浓度 以上时, 水中过量 DDTs 会向胶束中心的疏水假相集中, 从而提高疏水性 DDTs 在水中表观溶解度, 且随表面 活性剂浓度的升高而增加的趋势是非常快的 ${ }^{[64]}$.

\section{7 溶解性有机质}

溶解性有机质 (DOM) 是广泛存在于环境中由不同种类、复杂组分组成的混合物, 主要包括腐殖质与非 腐殖质类的有机物 ${ }^{[65]}$. DOM 是污染物迁移和转化的重要介质, 并且可能是化学物质长距离传输的载体. DOM 能与重金属和疏水性有机污染物等结合, 影响污染物在环境中的迁移、转化和分配等环境过程以及生 物性等.

DOM 具有丰富的一 $\mathrm{OH}$ 和一 $\mathrm{COOH}$ 等官能团, 它们能与金属离子结合从而改变其迁移性和生物累积性: 改变颗粒物对金属离子的吸附, 如 DOM 对 As 在沉积物颗粒上的吸附具有竞争作用 ${ }^{[66]}$; DOM 能与金属结合 成螯合物降低其自由溶解态浓度并影响其生物有效性, 如随着腐殖酸浓度越高, 水生无脊椎动物对可溶性 铅的摄取量越高 ${ }^{[67]}$. 当然, DOM 与金属的结合存在选择性, 即不同种类的 DOM 对不同的金属表现出不同的 亲合性,即不同的吸附、解吸能力, 同时 DOM 的存在形态也将直接影响金属的赋存形态.

有机污染物与 DOM 的结合会增加其在水中的溶解度, 促进了其在环境中的迁移. Gao 等 ${ }^{[10]}$ 研究由于沉 积物孔隙水中 DOC 的“增溶效应”, 使原位测定的杀虫剂 $K_{\mathrm{oc}}$ 值要远低于实验室平衡法得到的测定值, 说明 DOC 大大增加了水体中杀虫剂的含量, 增加了其再迁移能力. DOM 通常会降低有机污染物在水生生物体内 的生物富集 ${ }^{[68]}$, 而普遍认同的解释是只有自由溶解态的化学物质才能被生物富集, 与 DOM 结合产物的具有 过大的空间体积或过强的极性, 从而不能穿透生物膜而进人生物体内 ${ }^{[69]}$. 不过也有报道显示 DOM 在低浓 度时 (如小于 $10 \mathrm{mg} / \mathrm{L}$ ) 能够提高某些污染物的生物富集, 其可能的一种解释为当自由溶解态的 HOCs 被受 试生物损耗时, DOM 结合的有机污染物将暂时解吸出来而被生物吸收, 从而补偿有机污染物的耗损, 促进其 吸附动力学 ${ }^{[70]}$, 如 Mayer 发现多 DOM 条件下能促进有机污染物迁移扩散 ${ }^{[71]}$. 


\section{8 水动力条件}

对于湖泊、河流等水生态系统, 水体的水动力扰动 (如水流速度、风浪作用、船舶航运等) 会造成水体的 紊流扩散以及沉积物的再悬浮, 继而引起污染物在水体与沉积物之间的交换和迁移, 这种作用对浅水湖泊 尤为明显 ${ }^{[72]}$. 风浪作用和水底湍流会在沉积物一水界面产生垂向的剪切力, 较小的剪切力会增加污染物的 界面传质速率; 而大于临界值的剪切力会引起表层沉积物的再悬浮, 使吸附在悬浮颗粒物上的污染物由沉 积物的无氧环境进人上覆水中的有氧环境, 给水体造成严重的 “二次污染” ${ }^{[1]}$. Achman 等发现 Hudson 河口 区因沉积物再悬浮产生的 PCBs 负荷是废污水、大气沉降等外源输人量的 2 100 倍 ${ }^{[73]}$. 对沉积物 PCBs 模拟 释放研究, 以 $2.0 \mathrm{dyne} / \mathrm{cm}^{2}$ 大小剪切力作用于沉积物, 扰动状态下 PCBs 释放量相比静态对照组要高达上百 倍 ${ }^{[74]}$. 水底湍流一方面能快速输送污染物使之得到有效的稀释, 增加沉积物一水界面污染物的浓度梯度, 进 而产生较大的扩散驱动力; 同时, 较大的水底流速能显著减小 DBL 厚度, 即减少污染物在沉积物一水界面迁 移的传质阻力, 从而增强了界面污染物的扩散通量 ${ }^{[5]}$. Wang 等在获取原位实测数据进行拟合总结发现 DBL 厚度 $\delta_{\mathrm{DBL}}(\mathrm{mm})$ 与平均流速大小 $U(\mathrm{~mm} / \mathrm{s})$ 呈反比, 两者关系可以用 $\delta_{\mathrm{DBL}}=1686.1 D \cdot U^{-1}+0.1(D$ 为分子扩散 系数, $\left.\mathrm{mm}^{2} / \mathrm{s}\right)$ 来定量描述 ${ }^{[22]}$.

\section{9 生物扰动}

生物扰动 (bio-turbation) 主要是指底栖生物由于受食、潜穴、钻孔、躲避、分泌和排泄等活动对沉积物原 始结构和性质的改变, 进而影响沉积物中溶解态物质和颗粒物迁移转化的过程, 包括因底栖动物扰动造成 界面完整破坏的改造 (reworking) 以及对沉积物挖掘深穴的生物灌溉 (bio-irrigation) $)^{[75]}$. 一个小尺度 $(\mu \mathrm{m} \sim$ $\mathrm{m}$ ) 的生物扰动可以改变大尺度 (如 $50 \mathrm{~m} \sim 100 \mathrm{~km}$ ) 的沉积结构 ${ }^{[76]}$, 因此生物扰动构成了河口、近岸和浅海等 水体水层与底栖系统耦合过程的重要环节和枢纽 ${ }^{[77]}$.

生物扰动作用有多种影响因素, 如底栖动物种类 (体积、生物量等)、底栖动物密度、沉积物有机质含量、 沉积速率、水深和粒径等 ${ }^{[78-79]}$. 生物扰动可以直接对沉积物颗粒产生垂向上的混合和搬运、加速了孔隙水和 上覆水之间的物质交换、从而促进沉积物中的污染物向水体释放 ${ }^{[80-81]}$; 同时, 生物扰动还会引起沉积物一水 界面的溶解氧和微生物等变化, 进而间接改变污染物的扩散和转化 ${ }^{[82]}$. 例如, 向沉积物中添加摇蚊 (Chironomid larvae) 后, 对照组沉积物在 $4 \mathrm{~mm}$ 处溶解氧浓度降低至 0 , 而扰动处理组沉积物 $7 \mathrm{~mm}$ 处溶解氧浓度 仍可达到 $5 \mathrm{mg} / \mathrm{L}$, 且摇蚊的生物扰动能将总反硝化速率从 $0.76 \pm 0.34 \mathrm{mmol} \mathrm{N} /\left(\mathrm{m}^{2} \cdot \mathrm{d}\right)$ 提高到 $5.50 \pm 1.30$ $\mathrm{mmol} \mathrm{N} /\left(\mathrm{m}^{2} \cdot \mathrm{d}\right)^{[83]}$. 底栖生物扰动会加速沉积物中黏土物质的溶解, 孔隙水中营养盐扩散速率也会加 快 ${ }^{[84]}$, 如颤蚂的生物扰动提高氨氮从沉积物向水体的释放达到 $200 \%$ 、而溶解性活性磷 (SRP) 的释放速率也 提高了 $60 \%$ 以上 ${ }^{[85]}$.

生物扰动会改变沉积物中重金属、有机物等毒害污染物的吸附/解吸、分配/迁移等环境行为 ${ }^{[79,86]}$. 对重 金属而言, 生物扰动可改变沉积物中重金属的分布并促进重金属向上覆水释放, 如在悉尼港河口的研究发 现有生物扰动作用组中 $\mathrm{Zn}$ 的释放速率高达 $71 \mathrm{mg} /\left(\mathrm{m}^{2} \cdot \mathrm{d}\right)$, 是对照组的 2 倍以上 ${ }^{[87]}$; 改变重金属的赋存形 态, 如颤蚓的生物扰动提高沉积物中 $\mathrm{Cd}$ 的迁移能力, 并促进 $\mathrm{Cd}$ 从铁锰氧化物结合态向可交换态转移 ${ }^{[88]}$. 同样, 对于疏水性有机污染物而言, 生物扰动也能促进其从沉积物中释放, 研究发现挪威 Oslo 港底栖生物的 扰动可使沉积物中 PAHs、PCBs 和 DDTs 分别以 $243 、 19.6$ 和 $13.6 \mathrm{pmol} /\left(\mathrm{m}^{2} \cdot \mathrm{d}\right)$ 的速率向上覆水释放 ${ }^{[89]}$; 加 强有机物的富集和代谢 ${ }^{[90-91]}$, Pang 等发现由带丝蚓引起的生物扰动会显著增加沉积物中 PAHs 在生物体内 的累积, 同时对上覆水中的钩虾带来更大的毒性 ${ }^{[92]}$; 提高有机物的生物降解, 如 Timmermann 等通过室内研 究发现, 生物扰动组中萠的降解提高了 $180 \% \sim 200 \%{ }^{[93]}$, 这可能与生物扰动增加沉积物中的含氧量, 提高了 微生物降解有机污染物的能力有关 ${ }^{[94]}$.

\subsection{0 微生物}

沉积物一水界面是多种微生物参与, 并与物质发生频繁交换的有机综合体系, 该体系中微生物的活动可 以通过同化、异化作用来影响表层沉积物中有机质矿化途径和速率, 进而改变污染物的分布、形态以及迁移 转化等 ${ }^{[95]}$. 其中, 当外界环境条件的改变或营养物质的输人等可能会造成沉积物颗粒亚表面环境微生物群 落的变化, 并可能改变污染物的环境行为. 并对沉积物一水界面污染物的迁移转化产生重要影响 ${ }^{[06]}$.

沉积物一水界面附近的氧化还原条件直接影响到界面反应, 也决定了微生物的分布、活性以及其行为. 
表层沉积物一般呈氧化性,但水体溶解氧的穿透深度一般仅为几毫米; 而在深层沉积物中, 微生物对有机质 的降解会因合适的电子受体存在的情况下而进一步进行. 微生物对电子受体的选择一般需要该电子受体具 有合适的氧化还原电位 ${ }^{[97]}$, 沉积物一水界面按电子受体的难易程度通常为 $\mathrm{O}_{2} 、 \mathrm{NO}_{3}^{-} 、 \mathrm{Mn}^{4+} 、 \mathrm{Fe}^{3+} 、 \mathrm{SO}_{4}^{2-} 、 \mathrm{CO}_{2}$. 电子受体会因为其分布、有机质降解反应的吉布斯生成自由能的大小、以及微生物的异化还原能力的影响, 而在沉积物一水界面垂向上表现出一定顺序的分带, 即氧还原带、硝酸盐、还原带、锰还原带、铁还原带、硫酸 盐还原带和产甲烷带 ${ }^{[98]}$, 这一特点也决定了沉积物中一些功能微生物 ( 如厌氧氨氧化菌 ${ }^{[99]}$ 、录甲基化微生 物 ${ }^{[100]}$ 等) 对关键污染物迁移转化过程的调控.

微生物污染物有多种作用形式,以重金属为例,微生物与重金属之间可发生胞内、胞表及胞外等不同类 型之间的相互作用:胞内作用主要指对金属的吸收,比较重要的有生物甲基化过程;胞表相互作用包括了微 生物细胞表面与金属的结合、表面吸咐/沉降等;胞外相互作用包括分泌代谢物质改变外界环境、分泌有机 配合物 ${ }^{[101]}$. Ehrlich 认为只有原核生物 (含真细菌和古细菌) 才能在大尺度上对金属产生氧化还原作用, 改 变其形态, 并产生环境效应 ${ }^{[102]}$.

\subsection{1 其他因素}

除上环境因素外,还有其他的因素也会影响着污染物在沉积物一水界面的迁移、转化. 沉积物一水界面 的物质浓度梯度是其分子扩散的驱动力, 但界面扩散通量大小还受到传质量系数 ( $K_{\mathrm{m}}$, 传输阻力的倒数)、以 及物质在环境介质中扩散系数的影响 ${ }^{[103]}$. 当然, 污染物在沉积物一水界面的迁移行为是受到沉积物性质、 污染物性质、赋存形态以及多环境因子等多种耦合作用的结果 ${ }^{[1,104]}$, 在未来的研究中仍然要加强多因子耦 合作用下沉积物污染物迁移的定量化研究.

\section{5 沉积物一水界面污染物扩散通量的方法研究}

沉积物一水界面污染物扩散通量对评估污染物生物地球化学循环速率和水生态系统质量具有重要意 义. 首先, 扩散通量方向与大小是判断沉积物 “汇”或 “源”作用的重要指标. 其次, 扩散通量对于水生态系统 生态风险评价至关重要, 水生生物中污染物的累积程度与沉积物释放的量有关而非与沉积物中污染物的总 量相关. 最后, 扩散通量是用于污染沉积物修复的关键性参数, 能够用来评估原位沉积物修复的有效性. 例 如, 将材料覆盖在污染的沉积物表面, 可以通过估算沉积物一水界面扩散通量的变化来评价修复措施的效 果. 因此,有必要开展沉积物一水界面扩散通量的定量化研究. 估算沉积物一水界面污染物扩散通量主要有 4 种方法, 孔隙水浓度梯度法、实验室培养法、原位箱测定法和质量平衡法, 每种方法都有各自的特点 (表 1). 目前, 这些方法主要应用于营养盐、重金属等无机污染物扩散通量的计算, 针对有机污染物扩散通量的研究 也在逐步开展.

\section{1 孔隙水浓度梯度法}

沉积物一水界面的污染物浓度梯度是产生其界面扩散通量的直接驱动力, 孔隙水浓度梯度法通过测定 沉积物孔隙水和上覆水中溶解性污染物的浓度剖面分布, 结合菲克扩散第一定律 (公式 (1)) 估算污染物界 面扩散通量 ${ }^{[105]}$. 该方法的优点是操作简单易行, 工作量相对较小. 一般使用该方法时, 会假设界面污染物浓 度为线性变化的, 但实际条件下仅靠两点或有限的分层 (孔隙水、上覆水) 很难准确获取浓度梯度 $\partial c / \partial x$, 因 为计算值取决于采样分层尺度, 当分层尺度过大 (即垂向分辨率较低) 时, 很容易错过表层几厘米显著的浓 度梯度.

目前, 该方法多用于沉积物一水界面营养盐、重金属等无机污染物扩散通量估算. 环境样品中疏水性有 机物浓度相对低且样品基体复杂, 且其在沉积物一水界面通常具备较大的分配系数 $K_{\mathrm{p}}$, 因此, 直接通过分层 离心获取沉积物孔隙水并测定有机污染物的浓度相对困难 ${ }^{[9]}$. 同时, 野外获取的分配系数 $K_{\mathrm{p}}$ 可能因为沉积 物中碳质含量不同可能有数量级之间的差异. Peeper、DGT 等技术对无机物具有较好的效果, 对疏水性有机 污染物而言, 需要富集浓缩才能检出, 所以沉积物样品垂向分层不能做的太小 ( 因为要获取足够多样品来进 行有机物的富集), 从而导致无法准确获取界面附近的线性浓度变化梯度. 而不同研究者对线性部分斜率的 选取存在一定的主观性和任意性, 所以即使是相同的曲线,也可能会得到不同的结果, 而导致较大的偏差. 为此, 以膜材料为吸附相的被动采样技术研发并应用于测定沉积物一水界面有机污染物的扩散通量, 如 Cor- 
nelissen 等使用不同吸收材料 (聚甲醛 $55 \mu \mathrm{m} 、 500 \mu \mathrm{m}$, 聚二甲基硅氧烷 $200 \mu \mathrm{m}$ ) 组成的被动采样装置来测 定挪威Oslo 港口孔隙水和上覆水中自由溶解态 PAHs 浓度, 识别了沉积物的释放风险 ${ }^{[57]}$. Liu 等研发了多 段式沉积物孔隙水被动采样装置, 以低密度聚乙烯膜为吸附相, 获取高分辨率有机污染物在沉积物一水界面 的浓度分布曲线, 并基于数学模型推导, 计算出通过沉积物一水界面的污染物扩散通量 ${ }^{[14]}$, 很好地解决了上 述孔隙水浓度梯度法存在的问题.

\section{表 1 沉积物一水界面扩散通量研究方法优缺点比较}

Tab.1 Advantages and disadvantages of different diffusive fluxes methods across the sediment-water interface

\begin{tabular}{lll}
\hline 方法 & 优点 & 缺点 \\
\hline 孔 隙水浓度梯 & 相比其他 3 种方法,工作量小,操作比 & 忽略了生物扰动、水动力以及各种生化反应的影响. 传 \\
度法 & 较简单 & 统主动采样法的垂向分辨率不高,误差相对较大 \\
实验室培养法 & 常用,操作简单、方便,测定结果较为 & 无法克服水体中颗粒物的沉降补充和表层沉积物再悬 \\
& 准确 & 浮对物质扩散通量的影响,工作强度较大 \\
原位箱测定法 & 测定误差小,最接近实际 & 对配套条件需求较大,费用较高 \\
质量平衡法 & 适用于大范围水域物质扩散通量估算 & 上覆水物质的变化和传输很难准确确定,使用较少 \\
\hline
\end{tabular}

\section{2 实验室培养法}

由于孔隙水浓度梯度法使用条件和区域的局限性, 人们逐渐开始寻找新的测定方法, 实验室培养法成 为了最常用的估算方法之一. 从现场采集未受干扰、具有清晰的沉积物一水界面的沉积物柱状样,保持原样 不受破坏,运到实验室后在一定的培养条件下进行培养,通过上覆水中污染物浓度变化来计算其交换通量, 并分析如温度、溶解氧、pH 值、生物扰动等环境因素可能产生的影响 ${ }^{[106-107]}$. 根据研究者设计实验的目的,加 人上覆水的体积、沉积物量、培养时间、取样间隔等会有所差异. 在较短培养时间 (如小于 2 天) 时,沉积物一 水界面之间物质交换一般都达不到平衡状态, 随着培养时间的增加, 上覆水中溶解性污染物浓度变化会偏 离线性.

交换通量计算方法根据培养系统物质量守恒进行计算. 第 $i$ 个培养时间段 (每次取样即为 1 个培养时间 段)内污染物释放通量计算公式为:

$$
F_{i}=\left[Q_{i}-Q_{(i-1)}\right] /\left(A \cdot \Delta t_{i}\right)
$$

式中, $F_{i}$ 为第 $i$ 个培养时间段内物质通量, $i$ 为取样时间, 取值为 $1 、 2 、 3 、 4 、 \cdots$, , 般按天计算; $Q_{i}$ 为第 $i$ 次监测 培养管中物质含量, $Q_{0}$ 为培养开始时培养管中物质含量; $A$ 为沉积物一水界面面积; $\Delta t_{i}$ 为第 $i$ 个培养时间段 时长.

通过对培养管内上覆水污染物累积量和培养时间进行线性拟合得出线性拟合方程, 其斜率代表单位时 间培养管上覆水污染物累积量差值, 除以沉积物一水界面面积 (用培养管横截面代替) 即为培养过程中通量 平均值. 培养管中上覆水营养盐含量计算通式为:

$$
Q_{i}=C_{i} \cdot V_{1}+V_{2}\left(C_{1}+C_{2}+C_{3}+\cdots+C_{(i-1)}\right)-V_{2}\left(D_{1}+D_{2}+D_{3}+\cdots+D_{(i-1)}\right)
$$

式中, $i$ 取值为 $2 、 3 、 4 、 \cdots ; Q_{0}=C_{0} \cdot V_{1} ; Q_{1}=C_{1} \cdot V_{1} ; V_{1}$ 为培养管上覆水体积; $V_{2}$ 为每次取样体积; $C_{i}$ 为第 $i$ 次取样 测得的上覆水浓度值; $D_{i}$ 为第 $i$ 次取样测得的补充水浓度值.

由于室内培养管的体积有限, 一般该方法主要用于沉积物一水界面营养盐等无机污染物扩散通量的估 算. 实验室培养法的优点是简单、方便、测定结果比较准确, 而缺点则是无法克服现实水体中颗粒物的沉降 补充和表层沉积物的再悬浮对沉积物和水界面间物质交换速率和通量的影响, 而且有一定的工作强度.

\section{3 原位箱测定法}

原位箱测定法是将培养箱直接放置于水体沉积物表面, 围起一定面积的沉积物和上覆水, 用搅拌装置 使容器内上覆水保持均匀, 上部密封, 然后通过特定的采样口采样, 分析物质浓度的变化, 来计算沉积物一水 界面物质交换速率和通量 ${ }^{[108]}$. 同时可采用微电极测定培养箱内水体温度、溶解氧和 $\mathrm{pH}$ 值等环境因子的变 化. 扩散通量可由下式估算:

$$
F=M /(A \cdot t)
$$


式中, $M$ 为箱内积累污染物的量, $A$ 为吸收相与沉积物表面的接触面积, $t$ 为放置时间.

该方法与实验室培养法有着相似之处, 其优点是解决了实验室与现场条件的差异而引起的偏差, 且所 得结果是 4 种方法中最接近实际的, 缺点则是该方法的费用、技术要求、强度和难度都较高, 限制了其应用. 该方法曾经被广泛应用于营养盐、重金属等无机污染物通量的测定 ${ }^{[109]}$,近些年来被逐渐应用于疏水性有机 物扩散通量的测定 ${ }^{[13]}$. 不过该方法在测定沉积物一水界面有机污染物通量时也会存在一定偏差: 首先, 由于 原位箱置于一个封闭的沉积物表面, 长时间后会逐步形成缺氧甚至戻氧环境, 改变沉积物理化性质以及底 栖生物的活动, 对污染物的扩散造成干扰 ${ }^{[110]}$; 其次, 封闭体系下覆盖的沉积物没有受到水流的影响, 其沉积 物一水界面扩散边界层与自然水体有差别, 也会显著影响了界面污染物的扩散. 再者, 由于箱内存在吸附相, 该方法只能测定由沉积物向水中的扩散通量, 而无法识别沉积物 “汇” 的作用; 且吸附相对有机污染物具有 潜在的 “驱动力”, 加剧了沉积物中有机污染物向箱内扩散. 原位箱获取的通量为吸收相与水相之间的物质 通量, 与实际的沉积物一水界面扩散通量也存在一定偏差 ${ }^{[13]}$. 最后, 此方法只能获取时间 $t$ 内有机物的扩散 通量这一数值, 无法开展沉积物一水界面有机污染物其他环境行为的认识和研究.

\section{4 质量平衡法}

该方法通过测定水体上覆水污染物总输人和总输出, 基于物质质量平衡原理来估算沉积物一水界面污 染物的扩散通量. 该方法主要用于大范围水域营养盐释放量的估算, 由于上覆水中营养盐变化和传输难以 准确确定 ${ }^{[111]}$, 因此该方法工作量大、准确度相对较低,应用范围也比较小 ${ }^{[112]}$.

需要指出的是, 以上四种扩散通量估算方法均无法克服水底平流作用对通量的影响, 且各方法在应用 时均对污染物的扩散过程进行了简化处理. 事实上, 不同污染物由于其自身特性差异, 难以用统一的模型或 方法来开展相对准确的扩散通量研究. Dominic 编写专著 “Sediment flux modeling”对水体典型无机污染物 (氨氮、磷、硅、甲烷、钙、铁等) 在沉积物一水界面的扩散原理、扩散模型、定量分析、修正方法以及应用等方 面进行了详细的介绍 ${ }^{[113]}$, 可为沉积物一水界面无机污染物扩散通量估算研究提供良好的理论指导. 而在疏 水性有机污染物扩散通量方面,这方面的理论基础和技术研究还相对缺乏.

\section{6 研究展望}

沉积物一水界面是水环境中沉积物相和水相之间的过渡区, 对污染物的迁移、扩散以及转化具有重要的 作用. 当前人们对湖泊、水库、河流等水生生态系统的沉积物一水界面污染物的迁移扩散开展了大量的工作. 从发展趋势来看,未来该方向的研究还可从以下几个方面继续开展:

1) 扩大不同类型水体沉积物一水界面的关注范围. 沉积物一水界面具有显著的物理、化学和生物特征差 异, 是污染物发生迁移扩散、生化反应的“热区”. 未来研究中可开展多水体类型 (如深水/浅水湖泊、水库、河 流、湖滨带、河口、海湾、深海等), 多维度 (二维、三维) 或与多相界面 (大气一水以及土壤一大气) 结合的污染 物迁移扩散研究, 以完善水环境中污染物迁移转化的基本理论.

2) 深化多因素耦合作用下的沉积物一水界面污染物迁移扩散的认识. 污染物在沉积物一水界面的迁移 扩散是包括沉积物性质 (如孔隙结构、粒径分布、碳含量、有机质含量等)、环境因子(温度、氧化还原电位、水 动力等) 以及污染物特性 (吸附性、亲疏水性、生物富集性等) 共同作用的结果, 它们之间的相互关系错综复 杂. 而当前的研究仍以单要素和定性化作为为主, 多因素和定量化的研究还相对欠缺, 因此, 后续研究中需 要加强多因素耦合作用对沉积物一水界面污染物迁移扩散影响的研究.

3) 应用新技术新手段提升沉积物一水界面污染物迁移扩散过程的认识. 开展多学科交叉,结合新思路、 新技术, 如多污染物被动采样技术、多类别污染物同步检出技术、同位素示踪技术等, 为进一步探索污染物 的环境化学行为提供了技术支撑.

4) 推进沉积物一水界面污染物扩散通量与生物分析的融合, 为污染物生态毒理效应研究提供基础数据. 水生生物中污染物的累积程度与沉积物释放的量有关而非与沉积物中污染物的总量相关, 因此, 将沉积物一 水界面的污染物迁移扩散与生物分析进行融合, 提升多元复合污染体系下多种污染物综合生态效应的认 识,为水污染治理、生态修复以及人体健康提供依据.

5) 完善沉积物一水界面污染物扩散模型研究. 现场测定法的结果是最接近实际的, 但其研究条件要求 
和费用较高, 广泛应用还存在一定的困难. 而室内模拟实验不能充分说明现实环境中沉积物中污染物的迁 移转化过程. 数学模型是了解沉积物一水界面污染物迁移扩散过程的重要研究手段. 因此, 应完善特定污染 物, 特别是疏水性有机污染物的数学模型研究, 并与实验和观测相结合, 从而全面的评价沉积物中污染物对 水体的影响程度, 为环境监测部门和管理部门提供决策依据和科学支撑.

\section{7 参考文献}

[ 1 ] Dai GH, Liu XH. Factor affecting the migration of persistent organic pollutants across the sediment-water interface of aquatic environment. Environmental Chemistry, 2011, 30(1): 224-230. [戴国华, 刘新会. 影响沉积物一水界面持久性有 机污染物迁移行为的因素研究. 环境化学, 2011, 30(1): 224-230.]

[ 2 ] Hesslein RH. An in situ sampler for close interval pore water studies. Limnology and Oceanography, 1976, 21 (6) : 912-914.

[ 3 ] Mayer LM. Chemical water sampling in lakes and sediments with dialysis bags. Limnology and Oceanography, 1976, 21 (6) : 909-912.

[ 4 ] Gunnison D, Brannon JM, Jr IS et al. A reaction chamber for study of interactions between sediments and water under conditions of static or continuous flow. Water Research, 1980, 14(10) : 1529-1532.

[ 5 ] Gundersen JK, Jorgensen BB. Microstructure of diffusive boundary layers and the oxygen uptake of the sea floor. Nature, 1990, 345(6276) : 604-607.

[ 6 ] $\mathrm{Xu} \mathrm{D,} \mathrm{Wu} \mathrm{W,} \mathrm{Ding} \mathrm{S}$ et al. A high-resolution dialysis technique for rapid determination of dissolved reactive phosphate and ferrous iron in pore water of sediments. Science of the Total Environment, 2012, 422(4) : 245-252.

[ 7 ] Stahl H, Glud A, Schröder CR et al. Time-resolved pH imaging in marine sediments with a luminescent planar optode. Limnology and Oceanography Methods, 2006, 4(4) : 336-345.

[ 8 ] Stockdale A, Davison W, Zhang H. Micro-scale biogeochemical heterogeneity in sediments: A review of available technology and observed evidence. Earth Science Reviews, 2009, 92(1/2) : 81-97.

[ 9 ] Fabricius AL, Duester L, Ecker D et al. Metal and metalloid size-fractionation strategies in spatial high-resolution sediment pore water profiles. Environmental Science \& Technology, 2016, 50(17) : 9506-9514.

[10] Gao JP, Maguhn J, Spitzauer P et al. Distribution of pesticides in the sediment of the small Teufelsweiher pond ( southern Germany). Water Research, 1997, 31(11) : 2811-2819.

[11] McGroddy SE, Farrington JW, Gschwend PM. Comparison of the in situ and desorption sediment-water partitioning of polycyclic aromatic hydrocarbons and polychlorinated biphenyls. Environmental Science \& Technology, 1995, 30(1) : 172-177.

[12] Lick W. The sediment-water flux of HOCs due to " diffusion" or is there a well-mixed layer? If there is, does it matter? Environmental Science \& Technology, 2006, 40(18) : 5610-5617.

[13] Eek E, Cornelissen G, Breedveld GD. Field measurement of diffusional mass transfer of HOCs at the sediment-water interface. Environmental Science \& Technology, 2010, 44(17) : 6752-6759.

[14] Liu HH, Bao LJ, Zhang K et al. Novel passive sampling device for measuring sediment-water diffusion fluxes of hydrophobic organic chemicals. Environmental Science \& Technology, 2013, 47(17) : 9866-9873.

[15] Wang A. Studies on effects of the coexisted contaminants on the adsorption characteristics of atrazine onto the surficial sediments and their main components[Dissertation]. Changchun: Jilin University, 2009. [王乔. 共存污染物对沉积物及其 主要组分吸附阿特拉津的影响研究 [学位论文]. 长春: 吉林大学, 2009.]

[16] Qiao M, Qi W, Liu H et al. Simultaneous determination of typical substituted and parent polycyclic aromatic hydrocarbons in water and solid matrix by gas chromatography-mass spectrometry. Journal of Chromatography A, 2013, 1291 (4): 129-136.

[17] Zhang L, Wang Y, Sun C et al. Simultaneous determination of organochlorine, organophosphorus, and pyrethroid pesticides in bee pollens by solid-phase extraction cleanup followed by gas chromatography using electron-capture detector. Food Analytical Methods, 2013, 6(6): 1508-1514.

[18] Lei P, Zhang H, Shan BQ. A passive sampler with high resolution used for determining freely dissolved concentrations of contaminants in the sediment pore water. Chinese Patent: 201610130251.0, 2016. [雷沛, 张洪, 单保庆. 一种高分辨 测定孔隙水自由溶解态污染物浓度被动采样器, 专利申请号: 201610130251.0, 2016.] 
[19] Lei P, Shan BQ, Zhang H. Development and application for passive sampling techniques in aquatic environment, A review. Environmental Chemistry, 2018, 37(3) : 480-496. [雷沛, 单保庆, 张洪. 水体被动采样技术的发展与应用. 环 境化学, 2018, 37(3) : 480-496.]

[20] Fan CX, Zhou YY, Wu QL et al. The sediment-water interface of lakes: process and effects. Beijing: Science Press, 2013. [范成新, 周易勇, 吴庆龙等. 湖泊沉积物界面过程与效应. 北京: 科学出版社, 2013.]

[21] Boudreau BP, Jørgensen BB. The benthic boundary layer: transport processes and biogeochemistry, Oxford : Oxford University Press, 2001.

[22] Wang J, Zhao L, Wei H. Variable diffusion boundary layer and diffusion flux at sediment-water interface in response to dynamic forcing over an intertidal mudflat. Chinese Science Bulletin, 2012, 57(13) : 1568-1577.

[23] Reimers CE, Fischer KM, Merewether R et al. Oxygen microprofiles measured in situ in deep ocean sediments. Nature, 1986, 320: 741-744.

[24] Boudreau BP. The diffusive tortuosity of fine-grained unlithified sediments. Geochimica et Cosmochimica Acta, 1996,60 (16) : 3139-3142.

[25] Revsbech NP, Jørgensen BB. Advances in microbial ecology. Dordrecht, Netherlands: Springer, 1986.

[26] Davlson W, Zhang H. In situ speciation measurements of trace components in natural waters using thin-film gels. Nature, 1994, 367(6463) : 546-548.

[27] Ding SM, Han C, Wang YP et al. In situ, high-resolution imaging of labile phosphorus in sediments of a large eutrophic lake. Water Research, 2015, 74: 100-109.

[28] Devries CR, Wang FY. In situ two-dimensional high-resolution profiling of sulfide in sediment interstitial waters. Environmental Science \& Technology, 2003, 37(4) : 792-797.

[29] Motelica-Heino M, Naylor C, Zhang H et al. Simultaneous release of metals and sulfide in lacustrine sediment. Environmental Science \& Technology, 2003, 37(19): 4374-4381.

[30] Menegário AA, Lnm Y, Luko KS et al. Use of diffusive gradient in thin films for in situ measurements: A review on the progress in chemical fractionation, speciation and bioavailability of metals in waters. Analytica Chimica Acta, 2017, 983 : 54-66.

[31] Lewandowski J, Kristina RA, Hupfer M. Two-dimensional small-scale variability of pore water phosphate in freshwater lakes: Results from a novel dialysis sampler. Environmental Science \& Technology, 2002, 36(9) : 2039-2047.

[32] Ding S, Jia F, Xu D et al. High-resolution, two-dimensional measurement of dissolved reactive phosphorus in sediments using the diffusive gradients in thin films technique in combination with a routine procedure. Environmental Science \& Technology, 2011, 45(22) : 9680-9686.

[33] Gao Y, Sebastiaan VDV, Baeyens W. Two dimensional images of dissolved sulphide and metals in anoxic sediments by a novel DGT probe and optical scanning techniques. Trends in Analytical Chemistry, 2015, 66: 63-71.

[34] Wen SF. A methodology of sediment structure research and its application in Chaohu Lake[Dissertation]. Beijing: University of Chinese Academy of Sciences, 2013. [温胜芳. 一种沉积物结构研究的方法学构建及在巢湖的应用研究 [ 学位 论文]. 北京: 中国科学院大学, 2013.]

[35] Wang PB, Song JM, Guo ZY et al. The inducements of resuspention of sea surface sediment and their effects on recycle of biogenic elements. Marine Sciences, 2005, 29(10): 77-80. [王丕波, 宋金明, 郭占勇等. 海洋表层沉积物再悬浮的 诱因及其对生源要素循环的影响. 海洋科学, 2005, 29(10): 77-80.]

[36] Schwarzenbach RP, Gschwend PM, Imboden DM eds. Environmental organic chemistry. New York: Wiley, 2005.

[37] Iversen N, Jørgensen BB. Diffusion coefficients of sulfate and methane in marine sediments: Influence of porosity. Geochimica et Cosmochimica Acta, 1993, 57(3) : 571-578.

[38] Guenneau S, Puvirajesinghe TM. Fick's second law transformed: one path to cloaking in mass diffusion. Journal of the Royal Society Interface, 2013, 10(83): 1-6.

[39] Morgan EJ, Lohmann R. Detecting air-water and surface-deep water gradients of PCBs using polyethylene passive samplers. Environmental Science \& Technology, 2008, 42(19): 7248-7253.

[40] Piatt JJ, Backhus DA, Capel PD et al. Temperature-dependent sorption of naphthalene, phenanthrene, and pyrene to low organic carbon aquifer sediments. Environmental Science \& Technology, 1996, 30(3) : 751-760.

[41] Tremblay L, Kohl SD, Rice JA et al. Effects of temperature, salinity, and dissolved humic substances on the sorption of 
polycyclic aromatic hydrocarbons to estuarine particles. Marine Chemistry, 2005, 96(1) : 21-34.

[42] Seiki T, Izawa H, Date E. Benthic nutrient remineralization and oxygen consumption in the coastal area of Hiroshima Bay. Water Research, 1989, 23(2) : 219-228.

[43] Eckley CS, Hintelmann H. Determination of mercury methylation potentials in the water column of lakes across Canada. Science of the Total Environment, 2006, 368(1): 111-125.

[44] Todorova SG, Driscoll CT, Effler SW et al. Changes in the long-term supply of mercury species to the upper mixed waters of a recovering lake. Environmental Pollution, 2014, 185(4) : 314-321.

[45] Jonge MD, Teuchies J, Meire P et al. The impact of increased oxygen conditions on metal-contaminated sediments part I: effects on redox status, sediment geochemistry and metal bioavailability. Water Research, 2012, 46(7) : 2205-2214.

[46] Zoumis T, Schmidt A, Grigorova L et al. Contaminants in sediments : remobilisation and demobilisation. Science of the Total Environment, 2001, 266(1/2/3) : 195-202.

[47] Kelderman P, Osman AA. Effect of redox potential on heavy metal binding forms in polluted canal sediments in Delft (The Netherlands). Water Research, 2007, 41(18): 4251-4261.

[48] Belzile N, Chen YW, Gunn JM et al. Sediment trace metal profiles in lakes of Killarney Park, Canada: from regional to continental influence. Environmental Pollution, 2004, 130(2) : 239-248.

[49] Du LG, Rinklebe J, Vandecasteele B et al. Trace metal behaviour in estuarine and riverine floodplain soils and sediments: a review. Science of the Total Environment, 2009, 407(13) : 3972-3985.

[50] Jacobsen BN, Arvin E, Reinders M. Factors affecting sorption of pentachlorophenol to suspended microbial biomass. Water Research, 1996, 30(1) : 13-20.

[51] Gao JP, Maguhn J, Spitzauer P et al. Sorption of pesticides in the sediment of the Teufelsweiher pond ( Southern Germany). II: Competitive adsorption, desorption of aged residues and effect of dissolved organic carbon. Water Research, 1998, 32(7) : 2089-2094.

[52] Bo NJ, Arvin E, Reinders M. Factors affecting sorption of pentachlorophenol to suspended microbial biomass. Water Research, 1996, 30(1): 13-20.

[53] Luo XM, Liu CM. Effects of $\mathrm{Ca}^{2+}$ ionic strength on sorption of polycyclic aromatic hydrocarbons (PAHs) on soils and sediments in Yellow River Delta. Ecology and Environment, 2006, 15(5): 983-987. [罗雪梅, 刘昌明. 离子强度对土壤与 沉积物吸附多环芳烃的影响研究. 生态环境学报, 2006, 15(5): 983-987.]

[54] Means JC. Influence of salinity upon sediment-water partitioning of aromatic hydrocarbons. Marine Chemistry, 1995,51 (1) : 3-16.

[55] Rutherford DW, Chiou CT, Kile DE. Influence of soil organic matter composition on the partition of organic compounds. Environmental Science \& Technology, 1992, 26(2) : 336-340.

[56] Johnson WP, Amy GL. Facilitated transport and enhanced desorption of polycyclic aromatic hydrocarbons by natural organic matter in aquifer sediments. Environmental Science \& Technology, 1995, 29(3) : 807-817.

[57] Cornelissen G, Wiberg K, Broman D et al. Freely dissolved concentrations and sediment-water activity ratios of PCDD/Fs and PCBs in the open Baltic Sea. Environmental Science \& Technology, 2008, 42(23) : 8733-8739.

[58] Strom D, Simpson SL, Batley GE et al. The influence of sediment particle size and organic carbon on toxicity of copper to benthic invertebrates in oxic/suboxic surface sediments. Environmental Toxicology \& Chemistry, 2011, 30 ( 7 ): 1599-1610.

[59] Guven DE, Akinci G. Effect of sediment size on bioleaching of heavy metals from contaminated sediments of Izmir Inner Bay. Journal of Environmental Sciences, 2013, 25(9) : 1784-1794.

[60] Chiou CT, Kile DE. Deviations from sorption linearity on soils of polar and nonpolar organic compounds at low relative concentrations. Environmental Science \& Technology, 1998, 32(3) : 338-343.

[61] Yu Z, Huang W. Competitive sorption between $17 \alpha$-ethinyl estradiol and naphthalene/phenanthrene by sediments. Environmental Science \& Technology, 2005, 39(13) : 4878-4885.

[62] Ghosh R, Banerjee DK. Complexation of trace metals with humic acids from soil, sediment and sewage. Chemical Speciation \& Bioavailability, 1997, 9(1): 15-19.

[63] Li JH. Study on the effect of coexisting contaminants on sorption of three organic contaminants in soils/sediments[ Dissertation]. Shanghai : Shanghai Jiao Tong University, 2008. [李金花. 共存污染物对三种有机物在土壤/沉积物上吸附行 
为影响的研究 [学位论文]. 上海: 上海交通大学, 2008.]

[64] Kile DE, Chiou CT. Water solubility enhancements of DDT and trichlorobenzene by some surfactants below and above the critical micelle concentration. Environmental Science \& Technology, 1989, 23(7) : 832-838.

[65] Kerner M, Hohenberg H, Ertl S et al. Self-organization of dissolved organic matter to micelle-like microparticles in river water. Nature, 2003, 422(6928) : 150-154.

[66] Bauer M, Blodau C. Mobilization of arsenic by dissolved organic matter from iron oxides, soils and sediments. Science of the Total Environment, 2006, 354(2/3): 179-190.

[67] Sanchez-Marin P, Lorenzo JI, Blust R et al. Humic acids increase dissolved lead Bioavailability for marine invertebrates. Environmental Science \& Technology, 2007, 41(16) : 5679-5684.

[68] Urrestarazu RE, Meijer SN, Vaes WH et al. Using solid-phase microextraction to determine partition coefficients to humic acids and bioavailable concentrations of hydrophobic chemicals. Environmental Science \& Technology, 1998, 32(21): 3430-3435.

[69] Haitzer M, Höss S, Traunspurger W et al. Effects of dissolved organic matter ( DOM) on the bioconcentration of organic chemicals in aquatic organisms-A review. Chemosphere, 1998, 37(7) : 1335-1362.

[70] Nikkilä A, Paulsson M, Almgren K et al. Atrazine uptake, elimination, and bioconcentration by periphyton communities and Daphnia magna: effects of dissolved organic carbon. Environmental Toxicology and Chemistry, 2001, 20 ( 5 ): 1003-1011.

[71] Mayer P, Fernqvist MM, Christensen PS et al. Enhanced diffusion of polycyclic aromatic hydrocarbons in artificial and natural aqueous solutions. Environmental Science \& Technology, 2007, 41(17) : 6148-6155.

[72] Pang Y, Yan RR, Yu ZB et al. Suspension-sedimentation of sediment and release amount of internal load in Lake Taihu affected by wind. Environmental Science, 2008, 29(9) : 2456-2464. [逢勇, 颜润润, 余钟波等. 风浪作用下的底泥悬 浮沉降及内源释放量研究. 环境科学, 2008, 29(9) : 2456-2464.]

[73] Achman DR, Brownawell BJ, Zhang L. Exchange of polychlorinated biphenyls between sediment and water in the Hudson River estuary. Estuaries and Coasts, 1996, 19(4) : 950-965.

[74] Schneider AR, Porter ET, Baker JE. Polychlorinated biphenyl release from resuspended Hudson River sediment. Environmental Science \& Technology, 2007, 41(4) : 1097-1103.

[75] Crusius J, Bothner MH, Sommerfield CK. Bioturbation depths, rates and processes in Massachusetts Bay sediments inferred from modeling of ${ }^{210} \mathrm{~Pb}$ and ${ }^{239+240} \mathrm{Pu}$ profiles. Estuarine Coastal and Shelf Science, 2004, 61(4) : 643-655.

[76] Dietrich WE, Perron JT. The search for a topographic signature of life. Nature, 2006, 439(7075) : 411-418.

[77] Murray JMH, Meadows A, Meadows PS. Biogeomorphological implications of microscale interactions between sediment geotechnics and marine benthos: a review. Geomorphology, 2002, 47(1) : 15-30.

[78] Turnewitsch R, Witte U, Graf G. Bioturbation in the abyssal Arabian Sea: influence of fauna and food supply. Deep Sea Research Part II : Topical Studies in Oceanography, 2000, 47(14) : 2877-2911.

[79] Qin XB, Sun HW, Peng ST et al. Review of the impacts of bioturbation on the environmental behavior of contaminant in sediment. Acta Ecologica Sinica, 2014, 34(1):59-69. [覃雪波, 孙红文, 彭士涛等. 生物扰动对沉积物中污染物环 境行为的影响研究进展. 生态学报, 2014, 34(1):59-69.]

[80] Menone ML, Miglioranza KSB, Iribarne O et al. The role of burrowing beds and burrows of the SW Atlantic intertidal crab Chasmagnathus granulata in trapping organochlorine pesticides. Marine Pollution Bulletin, 2004, 48(3/4) : 240-247.

[81] Thibodeaux LJ, Bierman VJ. The bioturbation driven chemical release process. Environmetal Science \& Technology, 2003, 37( 13) : 252A-258A.

[82] Li YR. Effects of tubificid bioturbation on the physical and chemical characteristics of water-sediment interface[ Dissertation]. Changchun: Jilin University, 2016. [李耀睿. 颤蚓生物扰动对水一沉积物界面附近理化特征的影响 [学位论 文]. 长春: 吉林大学, 2016.]

[83] Shang J, Zhang L, Shi C et al. Influence of Chironomid larvae on oxygen and nitrogen fluxes across the sediment-water interface (Lake Taihu, China). Journal of Environmental Sciences, 2013, 25(5) : 978-985.

[84] Aller RC, Mackin JE, Ullman WJ et al. Early chemical diagenesis, sediment-water solute exchange, and storage of reactive organic matter near the mouth of the Changjiang, East China Sea. Continental Shelf Research, 1985, 4(1) : 227-251.

[85] Mermillodblondin F, Nogaro G, Vallier F et al. Laboratory study highlights the key influences of stormwater sediment 
thickness and bioturbation by tubificid worms on dynamics of nutrients and pollutants in stormwater retention systems. Chemosphere, 2008, 72(2): 213-223.

[86] Pang JX, You J. Influence of bioturbation on fate and bioavailability of sediment-associated contaminants. Asian Journal of Ecotoxicology, 2013, 8(6) : 805-816. [庞俊晓, 游静. 生物扰动对沉积物中污染物环境行为与生物可利用性的影 响. 生态毒理学报, $2013, \mathbf{8}(6): 805-816$.

[87] Simpson SL, Pryor ID, Mewburn BR et al. Considerations for capping metal-contaminated sediments in dynamic estuarine environments. Environmental Science \& Technology, 2002, 36(17) : 3772-3778.

[88] Lv JT, Hua XY, Dong DM et al. Effects of tubificid bioturbation on speciation of cadmium in contaminated sediment by laboratorial microcosm experiment. Journal of Jilin University:Science Edition, 2009,47( 5) : 1097-1103. [吕继涛, 花修 艺, 董德明等. 室内模拟研究颤蚓扰动对污染沉积物中镉形态分布的影响. 吉林大学学报: 理学版, 2009, 47(5): 1097-1103.]

[89] Schaanning M, Breyholtz B, Skei J. Experimental results on effects of capping on fluxes of persistent organic pollutants (POPs) from historically contaminated sediments. Marine Chemistry, 2006, 102(1/2) : 46-59.

[90] Nakata H, Sakai Y, Miyawaki T et al. Bioaccumulation and toxic potencies of polychlorinated biphenyls and polycyclic aromatic hydrocarbons in tidal flat and coastal ecosystems of the Ariake Sea, Japan. Environmental Science \& Technology, $2003,37(16): 3513-3521$.

[91] Goerke H, Weber K. Species-specific elimination of polychlorinated biphenyls in estuarine animals and its impact on residue patterns. Marine Eenvironmental Rresearch, 2001, 51(2) : 131-149.

[92] Pang J, Sun B, Li H et al. Influence of bioturbation on bioavailability and toxicity of PAHs in sediment from an electronic waste recycling site in South China. Ecotoxicology and Environmental Safety, 2012, 84(7) : 227-233.

[93] Timmermann K, Banta GT, Johnsen AR et al. Effects of the polychaetes Arenicola marina and Nereis diversicolor on microbial pyrene mineralization. Aquatic Microbial Ecology, 2008, 50( 2) : 197-207.

[94] Timmermann K, Banta GT, Klinge L et al. Effects of bioturbation on the fate of oil in coastal sandy sediments-An in situ experiment. Chemosphere, 2011, 82(10): 1358-1366.

[95] Wang FS, Liu CQ, Liang XB. Biogeochemical cycling of iron at lake water-sediment interface and its influence on trace metals. Geology-Geochemistry, 2003, 31(3) : 63-69. [汪福顺, 刘丛强, 梁小兵. 湖泊沉积物-水界面铁的微生物地球 化学循环及其与微量金属元素的关系. 地球与环境, 2003, 31(3): 63-69.]

[96] Papaspyrou S, Kristensen E, Christensen B. Arenicola marina (Polychaeta) and organic matter mineralisation in sandy marine sediments: In situ and microcosm comparison. Estuarine Coastal and Shelf Science, 2007, 72(1/2) : 213-222

[97] Nealson KH, Saffarini D. Iron and manganese in anaerobic respiration: environmental significance, physiology, and regulation. Annual Reviews in Microbiology, 1994, 48(1) : 311-343.

[98] Pakhomova SV, Hall POJ, Kononets MY et al. Fluxes of iron and manganese across the sediment-water interface under various redox conditions. Marine Chemistry, 2007, 107(3) : 319-331.

[99] Zhu G, Wang S, Wang W et al. Hotspots of anaerobic ammonium oxidation at land-freshwater interfaces. Nature Geoscience, 2013, 6(2): 103-107.

[100] Bravo AG, Bouchet S, Tolu J et al. Molecular composition of organic matter controls methylmercury formation in boreal lakes. Nature Communications, 2017, 8: 14255.

[101] Tang XY, Lv BS, Wu WH. Metal- microbe interaction in aquatic ecosystems. Research of Environmental Sciences, 1999, 12(3) : 28-30. [唐翔宇, 吕伯升, 吴文华. 水生生态系统中的微生物一金属相互作用. 环境科学研究, 1999, 12 (3) : 28-30.]

[102] Ehrlich HL. Microbes and metals. Applied Microbiology \& Biotechnology, 1997, 48( 6) : 687-692.

[103] Koelmans AA, Poot A, Lange HJD et al. Estimation of in situ sediment-to-water fluxes of polycyclic aromatic hydrocarbons, polychlorobiphenyls and polybrominated diphenylethers. Environmental Science \& Technology, 2010, 44 ( 8 ): 3014-3020.

[104] Liu HH, Bao LJ, Zeng EY. Recent advances in the field measurement of the diffusion flux of hydrophobic organic chemicals at the sediment-water interface. Trends in Analytical Chemistry, 2014, 54: 56-64.

[105] Lavery PS, Oldham CE, Ghisalberti M. The use of Fick's First Law for predicting porewater nutrient fluxes under diffusive conditions. Hydrological Processes, 2001, 15(13) : 2435-2451. 
[106] Wang PF, Yao Y, Wang C et al. Impact of macrozoobenthic bioturbation and wind fluctuation interactions on net methylmercury in freshwater lakes. Water Research, 2017, 124: 320-330.

[107] Zhu MY, Zhu GW, Wang YP. Influence of scum of algal bloom on the release of N and P from sediments of Lake Taihu. Environmental Science, 2011, 32(2) : 409-415. [ 朱梦圆, 朱广伟, 王永平. 太湖蓝藻水华衰亡对沉积物氮, 磷释放 的影响. 环境科学, 2011, 32(2): 409-415.]

[108] Cheng S, Liu H, Logan BE. Increased performance of single-chamber microbial fuel cells using an improved cathode structure. Electrochemistry Communications, 2006, 8 (3) : 489-494.

[109] Howell S. Intercalibration of benthic flux chambers I. Accuracy of flux measurements and influence of chamber hydrodynamics. Progress in Oceanography, 2004, 60(1): 1-28.

[110] Granberg ME, Gunnarsson JS, Hedman JE et al. Bioturbation-driven release of organic contaminants from Baltic Sea sediments mediated by the invading polychaete Marenzelleria neglecta. Environmental Science \& Technology, 2008, 42(4) : 1058-1065.

[111] Hammond DE, Fuller C, Harmon D et al. Temporal dynamics of an estuary: San Francisco Bay. Dordrecht, Netherlands: Springer, 1985 .

[112] Berelson WM, Heggie D, Longmore AD et al. Benthic nutrient recycling in Port Phillip Bay, Australia. Estuarine Coastal and Shelf Science, 1998, 46(6): 917-934.

[113] Dominic MD ed. Sediment flux modeling. New York: Wiley, 2001. 